\title{
Large eddy simulation of particle settling in the ocean mixed layer
}

Cite as: Phys. Fluids 18, 085109 (2006); https://doi.org/10.1063/1.2337098

Submitted: 04 December 2005 . Accepted: 17 July 2006 . Published Online: 29 August 2006

Y. Noh, I. S. Kang, M. Herold, and S. Raasch

\section{ARTICLES YOU MAY BE INTERESTED IN}

Vertical dispersion of light inertial particles in stably stratified turbulence: The influence of the Basset force

Physics of Fluids 22, 013301 (2010); https://doi.org/10.1063/1.3291678

Preferential concentration of particles by turbulence

Physics of Fluids A: Fluid Dynamics 3, 1169 (1991); https://doi.org/10.1063/1.858045

Equation of motion for a small rigid sphere in a nonuniform flow

The Physics of Fluids 26, 883 (1983); https://doi.org/10.1063/1.864230

\section{Scilight Highlights of the best new research} in the physical sciences 


\title{
Large eddy simulation of particle settling in the ocean mixed layer
}

\author{
Y. Noh ${ }^{\text {a) }}$ and I. S. Kang \\ Department of Atmospheric Sciences, Yonsei University, Seoul 120-749, Korea \\ M. Herold \\ Alfred Wegener Institute for Polar and Marine Research, 27570 Bremerhaven, Germany \\ S. Raasch \\ Institute of Meteorology and Climatology, University of Hannover, 30419 Hannover, Germany
}

(Received 4 December 2005; accepted 17 July 2006; published online 29 August 2006)

\begin{abstract}
The settling process of suspended particles in the turbulent flow was investigated by analyzing the Lagrangian motion of a large number of particles in the ocean mixed layer simulated by large eddy simulation (LES), focusing on the role of Langmuir circulation (LC). At the beginning, particles released near the surface are swept down rapidly following the downward jets in the presence of LC, contrary to the case of the horizontally uniform downward propagation in the absence of it. The strong vertical mixing by LC keeps the particle concentration almost uniform after the initial period in the presence of LC, while its mean concentration continues to decrease through particle settling. Under the influence of turbulence in the ocean mixed layer, the particle settling velocity $W$ is always smaller than $w_{s}$, and $W / w_{s}$ decreases with decreasing $w_{s} / u^{*}$, where $w_{s}$ is the terminal velocity of a particle in the still fluid and $u *$ is the frictional velocity. The presence of LC causes a further decrease of $W$ ultimately, and the difference between the cases with and without LC is the largest at $w_{s} / u * \sim 1$. The analysis of LES data reveals that particles spend more time in upward flows and that more particles tend to accumulate in the high vorticity region in the presence of LC. It suggests that particle trapping is more likely when the length scale of vortices is larger, which may lead to the decrease of $W$ in the presence of LC. It was also found that the preferential concentration occurs away from the regions of high horizontal vorticity and divergence when $w_{s} / u * \sim 1$. (C) 2006 American Institute of Physics. [DOI: 10.1063/1.2337098]
\end{abstract}

\section{INTRODUCTION}

The settling of biogenic particles from the ocean mixed layer to the deeper ocean provides an important mechanism for the vertical transfer of carbon in the ocean in that the particles carry with them the carbon absorbed through photosynthesis near the ocean surface, which is referred to as the biological pump. ${ }^{1,2}$ The carbon flux by the biological pump plays an important role in the global carbon cycle, crudely estimated as $10 \mathrm{Gt} \mathrm{C}$ per year, although the reliable estimation remains largely unknown. Being able to predict how suspended particles are dispersed and how long they stay within the ocean mixed layer before escaping to the deeper ocean is also important in the understanding of biologicalphysical interactions in the upper ocean. ${ }^{3}$

An accurate prediction of the particle settling process in the ocean mixed layer is thus imperative to understand the biogeochemical processes in the ocean. However, no information is available yet on how particles settle in the ocean mixed layer, and it is usually treated with the assumption that the particle settling velocity $W$ is the same as the terminal velocity in a still fluid, $w_{s}{ }^{4}$

There is much evidence contradicting this assumption, however. Stommel ${ }^{5}$ suggested that particles with no inertia can be suspended indefinitely, moving along closed trajectories, as a result of spending more time in upward flows, when

a)Electronic mail: noh@yonsei.ac.kr $w_{s}$ becomes sufficiently smaller than the characteristic fluid velocity $U$. This was later confirmed by Tooby et al. ${ }^{6}$ and Maxey and Corrsin ${ }^{7}$ by using a laboratory experiment and the numerical simulation of two-dimensional vortex flow, respectively.

Furthermore, Maxey and Corrsin $^{7}$ found that inertia, however weak, eventually causes all particles to settle, and the settling velocity of particles that are not trapped within the vortex is greater than $w_{s}$. The effect of a vortex on particle settling was also investigated recently by analyzing the trajectories of settling particles past an isolated vortex ${ }^{8,9}$ or the particle settling in random two-dimensional flow. ${ }^{10}$

Murray ${ }^{11}$ observed that the fall velocity is reduced as much as $30 \%$ from $w_{s}$ in laboratory experiments where turbulence is generated by grid oscillation. The reduction of settling velocity was also predicted in isotropic turbulence simulated by kinematic simulation. ${ }^{12}$ On the other hand, the settling velocity in isotropic turbulence was found to increase by the accumulation of particles in the low vorticity region and the consequent preferential sweeping of particles into downward moving fluid, when the inertial response time $\tau_{p}$ is comparable to the Kolmogorov time scale of turbulence $\tau_{k \cdot}{ }^{13-17} \mathrm{An}$ increase in the settling velocity also appears when the particle concentration is not uniform, caused by the inducement of virtual buoyancy. ${ }^{16,18-20}$

The ocean mixed layer has many unique characteristics, contrary to other turbulent boundary layers, because of the 
presence of the free surface. One of the most important phenomena is Langmuir circulation (LC), which is a collection of large circulation cells aligned along the wind direction induced by the interaction between the wind-driven surface shear and the Stokes drift of surface waves (see, e.g., Leibovich $^{21}$ ). Langmuir circulations are typically generated when the wind speed is greater than $3 \mathrm{~ms}^{-1}$, and its downward vertical velocity below the convergence region increases with the wind speed, sometimes exceeding $0.2 \mathrm{~ms}^{-1} \cdot{ }^{21-23}$ Surface convergence lines extend up to $2 \mathrm{~km}$ in length, and the spacing between cells ranges from two to hundreds of meters. The streaks are usually aligned downwind and propagate to the right of the main wind direction, implying the Coriolis effect.

The prevailing theory of LC is that of Craik and Leibovich, ${ }^{24}$ which describes the formation of LC in terms of an instability brought on by the interaction of the Stokes drift with the wind-driven surface shear current. The instability is initiated by an additional "vortex force" term in the momentum equation as $\boldsymbol{u}_{s} \times \boldsymbol{\omega}$, where $\boldsymbol{u}_{s}$ is the Stokes drift velocity and $\boldsymbol{\omega}$ is vorticity.

All previous works on the effect of a vortex on particle settling have considered only a stationary two-dimensional vortex. ${ }^{5-10}$ It is therefore interesting from the fluid dynamical point of view how the presence of a realistic large-scale coherent vortex in the fluctuating turbulent flow such as LC affects the particle settling process.

The development of an appropriate large eddy simulation (LES) model for the ocean mixed layer has been hindered, compared to other turbulent boundary layers, by the difficulty of handling the boundary condition at the sea surface, in which wave breaking and LC are present. Skyllingstad and Denbo ${ }^{25}$ and McWilliams et al. ${ }^{26}$ successfully reproduced LC in the LES of the ocean mixed layer in which the momentum equation is modified by including a vortex force and an additional advection by the Stokes drift, following the theory by Craik and Leibovich. ${ }^{24}$ Recently, Noh et $a l .^{27}$ further elaborated the LES model of the ocean mixed layer by incorporating the effects of wave breaking as well as LC. Successful simulations of the motion of suspended particles in turbulent flows by using LES have been accomplished recently in various fields. ${ }^{28-33}$ Meanwhile, a direct comparison between the motion of Lagrangian floats in the ocean and LES results was attempted by Harcourt et al. ${ }^{34}$

In the present paper, we attempt to understand the settling process of suspended particles introduced into the ocean mixed layer through the sea surface by analyzing the Lagrangian motion of a large number of particles in the turbulent flow field generated by LES. We investigate how the settling process is affected by the characteristics of the turbulence structure of the ocean mixed layer and of particles. We predict how long particles are sustained in the ocean mixed layer before escaping to the deep ocean.

\section{LES MODEL AND SIMULATIONS}

The LES model we used in this study was developed based on the PALM (PArallelized LES Model), which has been applied extensively to the atmospheric boundary
TABLE I. Summary of experimental conditions.

\begin{tabular}{cccccccc}
\hline \hline \multicolumn{3}{c}{ Fluid } & & & \multicolumn{2}{c}{ Particle } \\
\cline { 1 - 1 } \cline { 6 - 7 } EXP & $U_{s}\left(\mathrm{~ms}^{-1}\right)$ & EXP & $u^{*}\left(\mathrm{~ms}^{-1}\right)$ & & EXP & $w_{s}\left(\mathrm{~ms}^{-1}\right)$ \\
\hline $\mathrm{O}$ & 0 & $\mathrm{~A}$ & 0.01 & & \\
& & $\mathrm{~B}$ & 0.013 & & $\alpha$ & $10^{-3}$ \\
$\mathrm{~L}$ & \multirow{2}{*}{0.049} & $\mathrm{C}$ & 0.005 & & $\beta$ & $10^{-2}$ \\
& & $\mathrm{D}^{\mathrm{a}}$ & 0.01 & & $\gamma$ & 0 \\
\hline \hline
\end{tabular}

${ }^{\mathrm{a}} \mathrm{A}$ homogeneous mixed layer without a thermocline.

layer, ${ }^{35-38}$ the ocean deep convection, ${ }^{39,40}$ and the ocean mixed layer. ${ }^{27,41}$ Subgrid-scale turbulence, which is modeled according to Deardorff, ${ }^{42}$ is widely used in the LES of geophysical turbulence. ${ }^{43-45}$ A prognostic equation is solved for the subgrid-scale turbulent kinetic energy (TKE), which is used to parametrize the subgrid-scale fluxes. The performance of the parallelized code is found to be excellent with an almost linear speed-up to a very large number of processors. ${ }^{46}$

For the application to the ocean mixed layer, several modifications were made. A free slip boundary condition was imposed at the surface. The momentum equation was modified by including a vortex force and an additional advection by the Stokes drift following the theory by Craik and Leibovich. ${ }^{24-27}$ The effect of wave breaking was not considered here, since we are concerned mainly with the particle settling process below the near-surface zone.

The box-filtered equation over the grid size of LES is then represented as

$$
\begin{aligned}
\frac{\partial u_{i}}{\partial t}+\left(u_{j}+u_{s j}\right) \frac{\partial u_{i}}{\partial x_{j}}= & -\frac{1}{\rho_{0}} \frac{\partial p}{\partial x_{i}}-\varepsilon_{i j k} f_{j}\left(u_{k}+u_{s k}\right)+\varepsilon_{i j k} u_{s j} \omega_{k} \\
& -\frac{\partial}{\partial x_{j}} \tau_{i j}
\end{aligned}
$$

where $\tau_{i j}$ is the subgrid stress tensor.

For simplicity, we assumed that both wind stress and wave field are in the $x$ direction, and further that the wave field is steady and monochromatic. The associated Stokes velocity is then given by

$$
u_{s}=U_{s} \exp (-4 \pi z / \lambda)
$$

with $U_{s}=(\pi a / \lambda)^{2}(g \lambda / 2 \pi)^{1 / 2}$, where $a$ is the wave height, $\lambda$ is the wavelength, and $g$ is gravitational acceleration. For wave height and wavelength, we used typical values such as $a=1.0 \mathrm{~m}$ and $\lambda=40 \mathrm{~m}$, which makes $U_{s}=0.049 \mathrm{~ms}^{-1}$.

The model domain was $300 \mathrm{~m}$ in the horizontal direction $(x$ and $y)$ and $80 \mathrm{~m}$ in the vertical direction $(z)$. The number of grid points was $240 \times 240 \times 64$, and the corresponding grid sizes were $1.25 \mathrm{~m}$ in both horizontal and vertical directions. The Coriolis force was given by $f=1.2 \times 10^{-4} \mathrm{~s}^{-1}$. The horizontal boundaries were periodic, and a radiative free slip boundary condition was applied at the bottom.

McWilliams et al. $^{26}$ suggested the turbulent Langmuir number $\mathrm{La}_{t}$ that characterizes the relative importance of LC in the ocean mixed layer, given by 

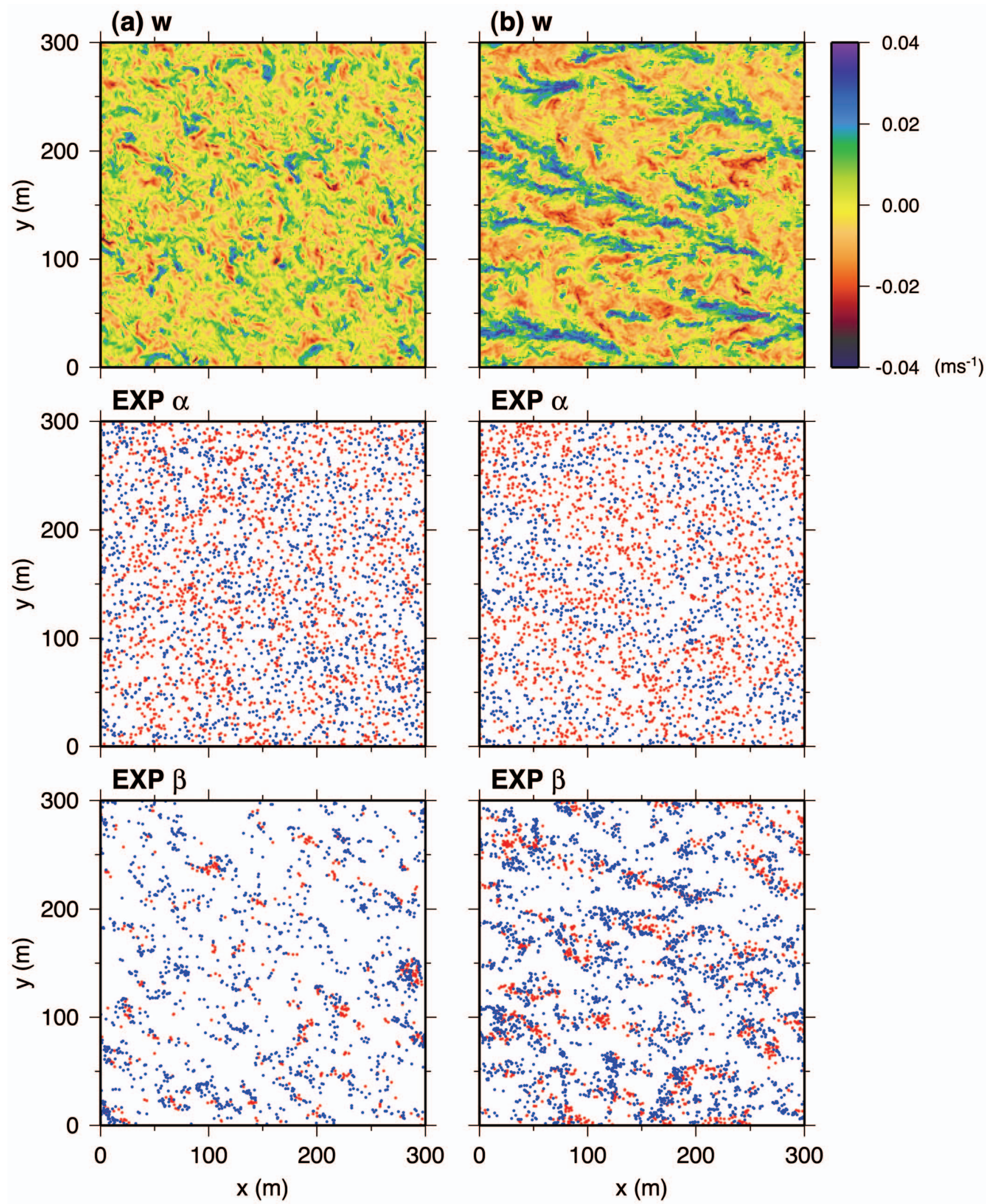

FIG. 1. (Color) Instantaneous distributions of vertical velocity (top), particles of EXP $\alpha\left(w_{s}=10^{-3} \mathrm{~ms}^{-1}\right)$ (middle) and of EXP $\beta\left(w_{s}=10^{-2} \mathrm{~ms}^{-1}\right)($ bottom) at the horizontal cross sections $(z=10 \mathrm{~m})$ at $t^{*}=0.4$. Here the red and blue dots represent the particles with upward and downward vertical velocities, respectively; (a) EXP OA, (b) EXP LA. 

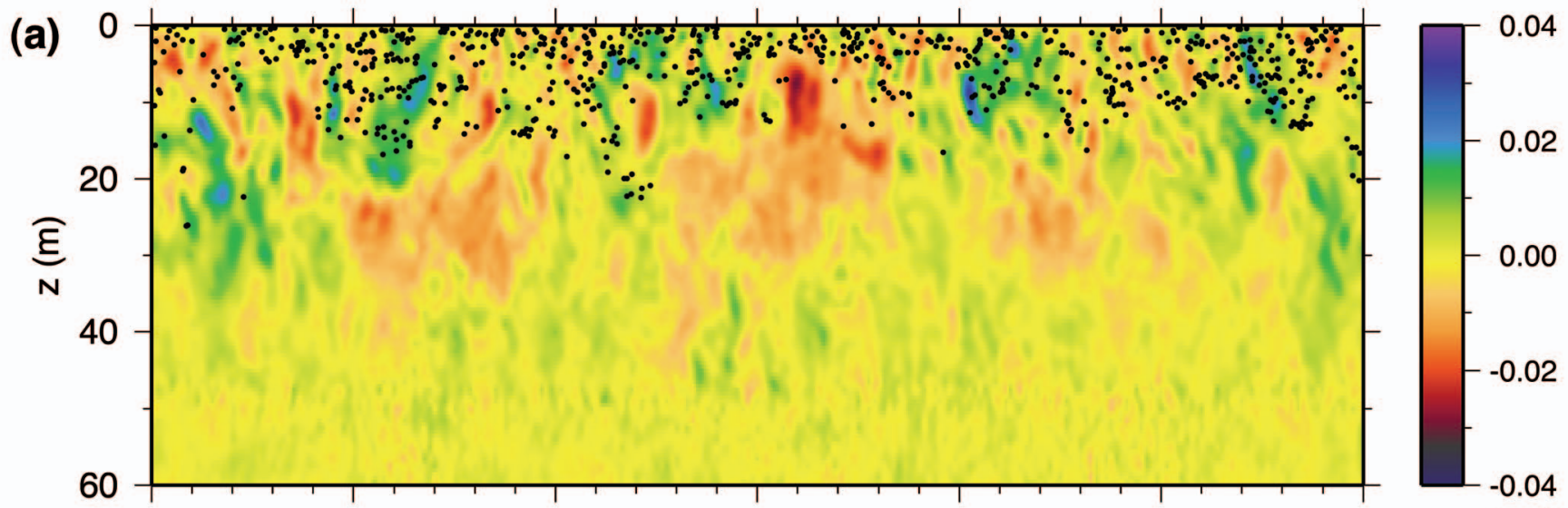

$\left(\mathrm{ms}^{-1}\right)$

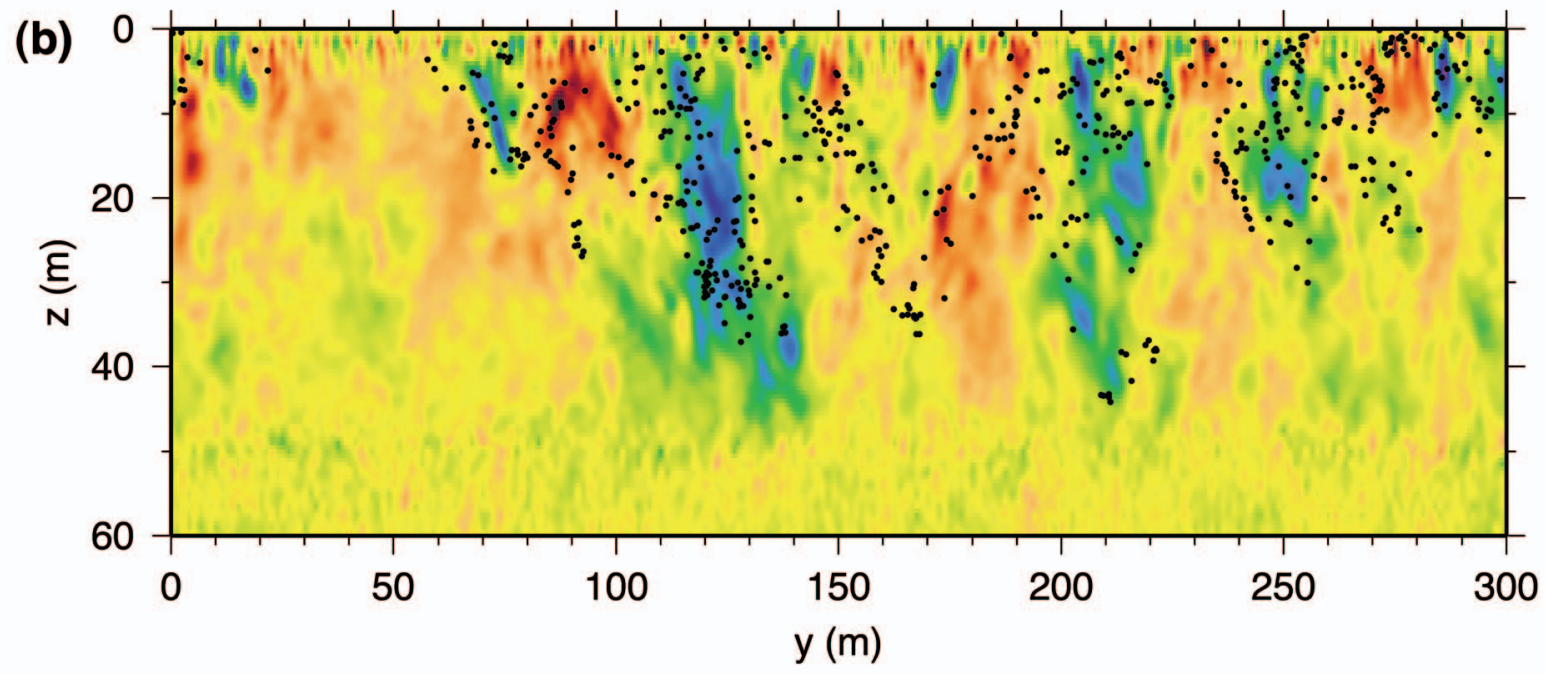

FIG. 2. (Color) Instantaneous distributions of particles and vertical velocity at the vertical cross section at $t^{*}=0.04$; (a) EXP OA_ $\alpha$, (b) EXP LA_ $\alpha$.

$$
\mathrm{La}_{t}=\left(u_{*} / U_{s}\right)^{1 / 2},
$$

where $u_{*}$ is the frictional velocity due to wind stress. The conditions of the present simulation lead to $\mathrm{La}_{t}=0.45$, which is consistent with the typical values of $\mathrm{La}_{t}$ such as $\mathrm{La}_{t}$ $\sim 0.2-0.5$ under quasiequilibrium conditions of wind and waves. ${ }^{47}$ In the present paper, we considered only the case with no surface heat flux.

If a particle is sufficiently small $\left[\operatorname{Re}_{p}(=d|\boldsymbol{u}-\boldsymbol{V}| / \nu) \ll 1\right]$, the equation, which determines the instantaneous velocity $\boldsymbol{V}$ of a suspended particle, is given by

$$
\frac{d V_{i}}{d t}=\frac{\beta}{\beta+C_{M}} \frac{1}{\tau_{p}}\left(u_{i}-V_{i}-w_{s} \delta_{i 3}\right)+\frac{1+C_{M}}{\beta+C_{M}} \frac{d u_{i}}{d t} .
$$

Here $\tau_{p}$ is the inertial response time, $C_{M}$ is the added mass coefficient (equal to $1 / 2$ ), and $\beta$ is the ratio of densities of a particle and fluid, defined by $\beta=\rho_{p} / \rho_{f}$, where $\rho_{p}$ and $\rho_{f}$ represent the particle and fluid density, respectively.

The inertial response time $\tau_{p}$ and the terminal velocity in the still fluid $w_{s}$ are given by

$$
\tau_{p}=\frac{\beta d^{2}}{18 \nu}
$$

and

$$
w_{s}=\frac{g(\beta-1) d^{2}}{18 \nu}
$$

for $\operatorname{Re}_{p} \ll 1$, where $d$ is the diameter of a particle and $\nu$ is the kinematic viscosity of a fluid.

If $d$ and $\beta-1$ of a particle are of orders of $1-100 \mu \mathrm{m}$ and $0.1-1$, respectively, as is typical for particles observed in the ocean, ${ }^{2}$ then $w_{s}$ and $\tau_{p}$ are estimated as $w_{s}$ $\sim 10^{-8}-10^{-2} \mathrm{~ms}^{-1}$ and $\tau_{p} \sim 10^{-8}-10^{-3} \mathrm{~s}$, respectively. Meanwhile, the Kolmogorov time and length scales, $\tau_{k}\left[=(\nu / \varepsilon)^{1 / 2}\right]$ and $\eta\left[=\left(\nu^{3} / \varepsilon\right)^{1 / 4}\right]$, can be estimated as $\tau_{k}$ $\sim 0.1-10 \mathrm{~s}$ and $\eta \sim 10^{-3} \mathrm{~m}$, since the value of $\varepsilon$ is in the range $\left(2 \times 10^{-8}\right)-\left(5 \times 10^{-5}\right) \mathrm{m}^{2} \mathrm{~s}^{-3}$ in the simulated ocean mixed layer. ${ }^{27}$ Based on these estimations, we chose the appropriate values of parameters as $w_{s}=10^{-2}$ and $10^{-3} \mathrm{~ms}^{-1}$ and the Stokes number St $\left(=\tau_{p} / \tau_{k}\right)=10^{-4}$, by assuming that $\tau_{p}=10^{-4} \mathrm{~s}$ and $\tau_{k}=1 \mathrm{~s}$.

The very small length and time scales of a particle, relative to those of the fluid flow, justify the neglect of lift and shear effects in (4). ${ }^{8,9}$ Moreover, since $\mathrm{St}=10^{-4}$, the deviation from the case of no inertia such as 


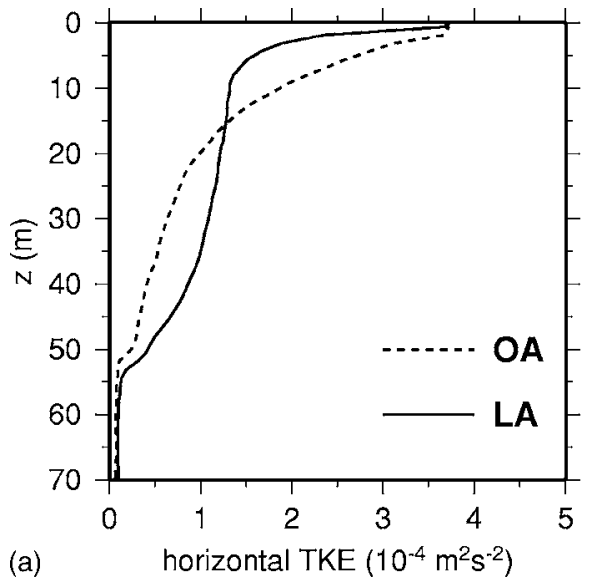

(a)

$$
V_{i}=u_{i}-w_{s} \delta_{i 3}
$$

becomes insignificant, which is usually the case for small particles suspended in the ocean. ${ }^{33,48,49}$ It also implies that the argument on the trapping of particles in a vortex motion, proposed by Stommel, ${ }^{5}$ can be relevant in the present case.

We considered only the resolved velocity field for $u_{i}$ similarly to other LES experiments, ${ }^{28-33}$ because the subgrid scale TKE is negligibly small through the whole depths of the ocean mixed layer, as shown in Noh et al. ${ }^{27}$

We investigated the motions of settling particles for two different types of the ocean mixed layer; that is, with and without LC (EXP O and EXP L, respectively). For each case, we carried out simulations with different wind stresses. EXP A is the control experiment with $u *=0.01 \mathrm{~ms}^{-1}$, which corresponds to the wind velocity $5-10 \mathrm{~ms}^{-1}$ at $10 \mathrm{~m}$ above sea level. EXP B and $C$ represent the cases with stronger and weaker wind stresses $\left(u *=0.013\right.$ and $\left.0.005 \mathrm{~ms}^{-1}\right)$, respectively. In addition, we investigated the case of a homogeneous mixed layer in order to investigate the effects of a finite depth of the mixed layer bounded by a thermocline (EXP D). In EXP D, the wind stress is the same as in EXP A.

For a given flow type, we investigated the motions of two different types of particles, that is, with slow and fast settling velocities, represented by EXP $\alpha\left(w_{s}=10^{-3} \mathrm{~ms}^{-1}\right)$ and $\operatorname{EXP} \beta\left(w_{s}=10^{-2} \mathrm{~ms}^{-1}\right)$. We also performed the simulation for the case of a passive tracer for comparison (EXP $\gamma$ ). Henceforth, we will refer to a specific experiment by combining three letters representing the characteristics of the flow and particles, e.g., EXP OA_ $\alpha$. Experimental conditions are summarized in Table I.

Integration was carried out without particles for $8 \mathrm{~h}$ until equilibrium was reached. The initial thickness of the ocean mixed layer of uniform density was given by $h=50 \mathrm{~m}$, which was bounded below by strong stratification $\left(N^{2}=10^{-4} \mathrm{~s}^{2}\right)$ (except EXP D). After the flow field approached equilibrium, 88000 particles were released in the near surface level $(z$ $=2.5 \mathrm{~m}$ ) with uniform horizontal distribution. We define this moment as the initial time, i.e., $t=0 \mathrm{~s}$. The integration was then continued further until $t^{*}\left[\equiv t /\left(h / w_{s}\right)\right]=2$. In analyzing the result hereafter, time is rescaled by $h / w_{s}$. Note that all particles must settle below $h$ at $t^{*}=1$ in the still fluid. During the experiments, the entrainment rate of the mixed layer depth, $w_{e} \sim 10^{-5} \mathrm{~ms}^{-1}$, was much smaller than $w_{s}$, and the stratification within the mixed layer induced by the entrainment at the bottom of the mixed layer was negligible $\left(N^{2}\right.$ $\sim 10^{-8} \mathrm{~s}^{-1}$ ).

\section{RESULTS}

\section{A. Characteristics of the ocean mixed layer simulated by LES}

In order to analyze the motion of particles, we first need to understand the structure of turbulent flows in the ocean mixed layer simulated by LES. Figure 1 shows the distributions of vertical velocity and particles at the horizontal cross sections at $t^{*}=0.4$ for the cases with $u *=0.01 \mathrm{~ms}^{-1}$ (EXP OA and EXP LA). It also presents the distribution of particles at the corresponding cross sections, which will be discussed in the next section.
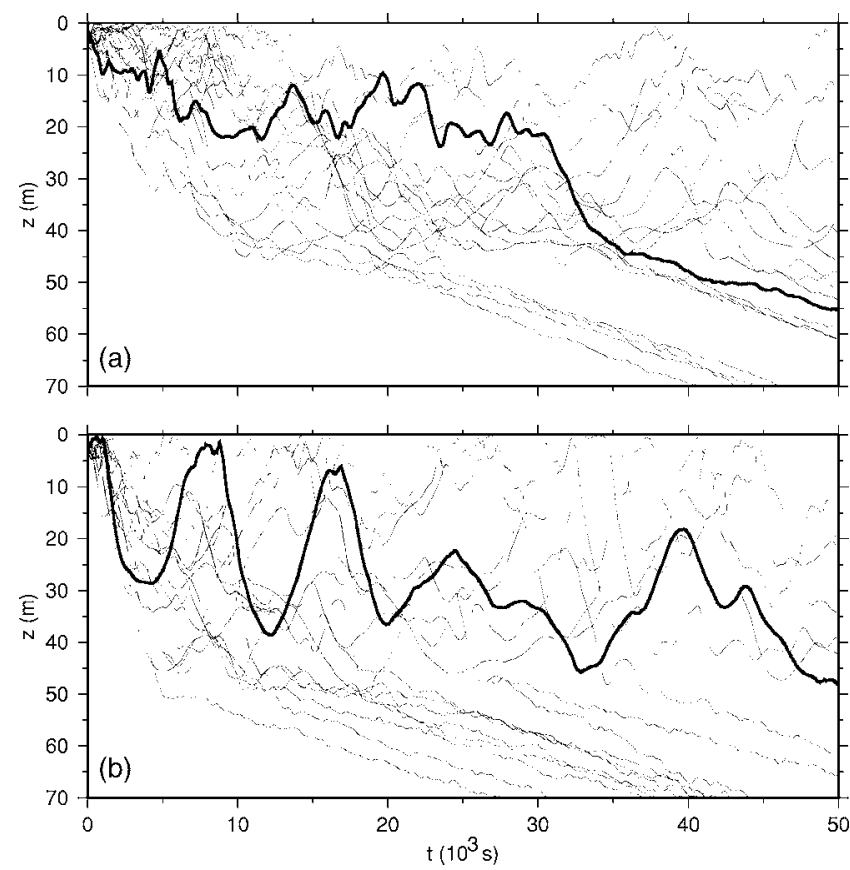

FIG. 4. Tracks of the vertical positions of particles. One typical track is shown by a thick line in each figure; (a) EXP OA_ $\alpha$, (b) EXP LA_ $\alpha$. 

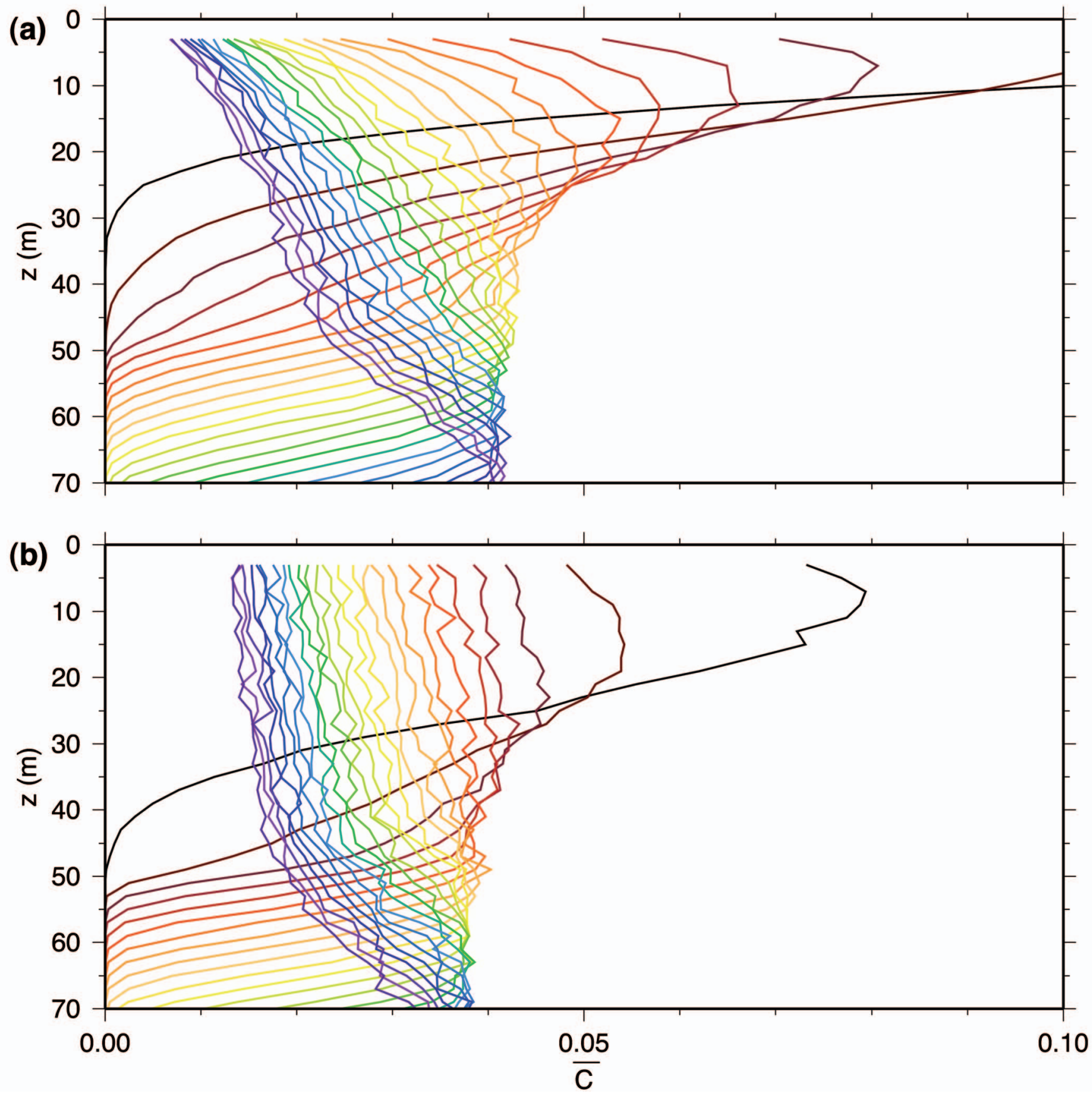

FIG. 5. (Color) Evolutions of the mean concentration profiles with time $\left(\Delta t^{*}=0.04\right)$; (a) EXP OA_ $\alpha$, (b) EXP LA_ $\alpha$.

The effects of LC are shown clearly in the comparison of the cases EXP OA and EXP LA. In the presence of the vortex force (EXP LA), streaks of the strong downwelling zone beneath the surface convergence appear in parallel to each other, associated with the formation of Langmuir cells. It was also found that the stripes are longitudinally oriented along the direction of wind stress near the surface, but the direction of stripes spirals clockwise toward a diagonal orientation with increasing depth due to the Coriolis effect, as shown in Noh et $a .^{27}$ The downwelling velocity field is narrower and stronger than the upwelling one. The downward velocity increases typically up to $0.04 \mathrm{~ms}^{-1}$. The distance between the stripes increases with depth, reaching about $100 \mathrm{~m}$ at $z$
$=25 \mathrm{~m}$. Instances of joining of convergence zones with $\mathrm{Y}$ junctions appear, as observed in the ocean. ${ }^{50,51}$

On the other hand, in the absence of a vortex force (EXP OA), no organized structure appears, and the velocity field is more or less isotropic without any directional tendency. The downward velocity is weaker, and the upwelling and downwelling velocity fields are rather symmetrical. It was also found that the length scale of eddies increases with depth, which is consistent with the assumption used in most ocean mixed layer models. ${ }^{52,53}$

The corresponding distributions of the vertical velocity and particles at the vertical cross section are shown in Fig. 2, although we showed the pictures at $t^{*}=0.04$ here in order to 


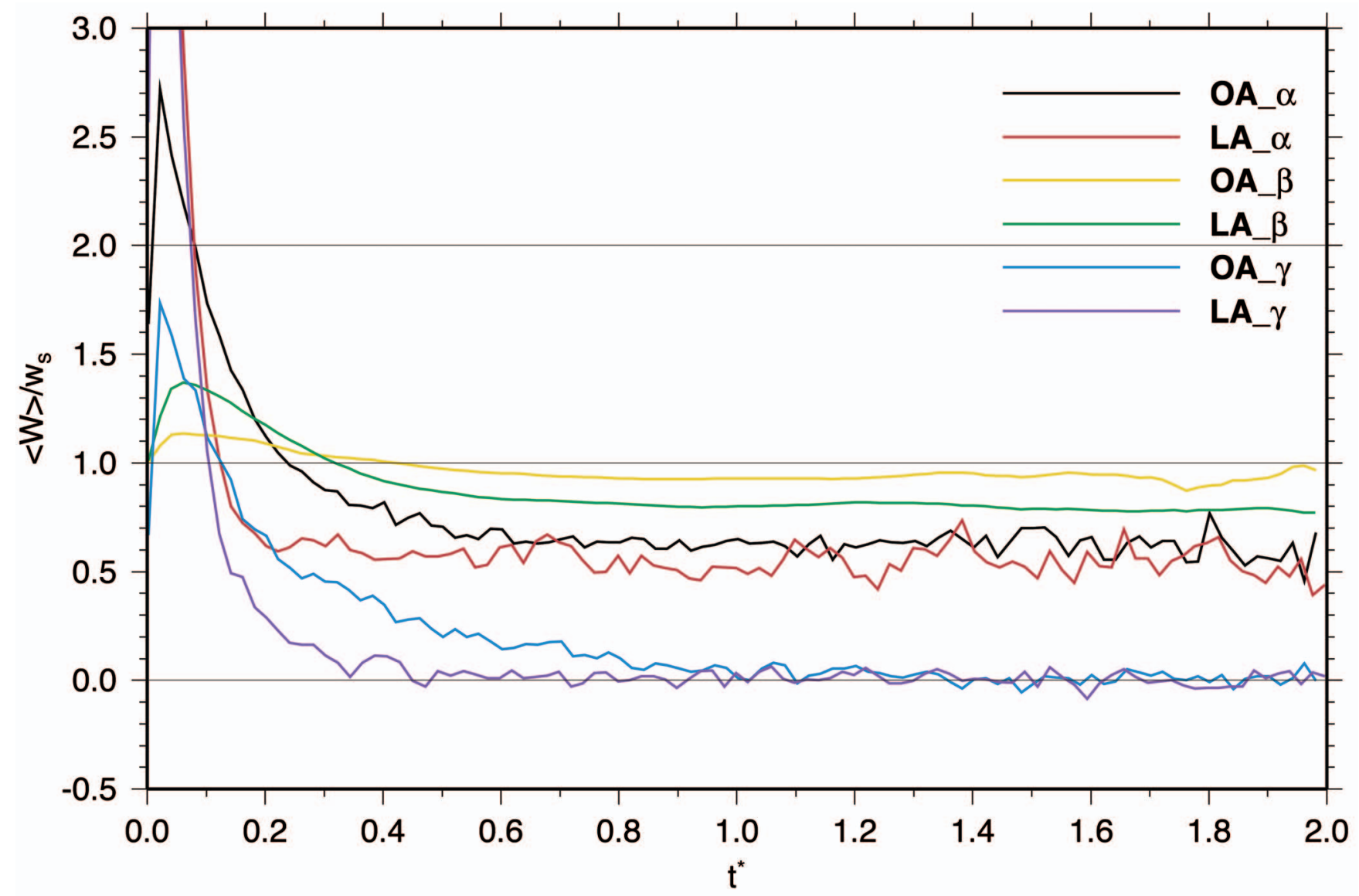

FIG. 6. (Color) Variation of the mean vertical velocity $\langle W\rangle$ of particles with time $t^{*}\left[=t /\left(h / w_{s}\right)\right]$ for each experiment. In the case of a passive tracer, both time and velocity scales were rescaled by using $w_{s}=10^{-3} \mathrm{~ms}^{-1}$.

capture the early stage of the downward propagation of particle settling (see Sec. III B). In the case of EXP LA, strong downward jets associated with LC are clearly observed within the mixed layer.

The effects of LC in the structure of the ocean mixed layer are reflected in the vertical profiles of the mean horizontal and vertical turbulent kinetic energies (TKE), where the horizontal mean profiles from LES were obtained by averaging over $600 \mathrm{~s}$ (Fig. 3).

A significant increase in the vertical TKE is observed over the entire depth of the ocean mixed layer in the presence of LC (EXP LA). This reflects the influence of the strong downward jets associated with LC, as shown in Fig. 1. In addition, the horizontal TKE is decreased in the upper layer in the range of $z=5-15 \mathrm{~m}$, when it is influenced by LC. Very effective vertical mixing of momentum down to much deeper depths in EXP LA also causes the velocity shear almost to disappear in the presence of LC (EXP LA), as shown in Noh et al., ${ }^{27}$ and thus results in a reduced shear production. It reveals the dominant role of LC in the vertical mixing process in the ocean mixed layer. The low-level TKE appearing below the mixed layer is due to the downward propagation of internal gravity waves.

The flow patterns and the characteristics of the mean profiles reproduced in the present simulation are in good agreement with the previous LES results. ${ }^{25,26}$ One can also refer to Noh et $a l^{27}$ for a detailed description of the flow field.

\section{B. Pattern of particle settling}

Distributions of particles in the horizontal cross sections in the flow field from EXP OA and EXP LA are shown in Fig. 1 for the cases of EXP $\alpha\left(w_{s}=10^{-3} \mathrm{~ms}^{-1}\right)$ and $\operatorname{EXP} \beta$ $\left(w_{s}=10^{-2} \mathrm{~ms}^{-1}\right)$. They are obtained from particles located within the thickness of $2.5 \mathrm{~m}$ from the corresponding cross sections. Here the red and blue dots represent the particles with upward and downward vertical velocities, respectively.

Close correlations are observed between the vertical velocities of fluid and particles in the case of $\operatorname{EXP} \alpha$ reflecting the very small particle inertia in the present simulation; see, e.g., the streak pattern along LC in EXP LA_ $\alpha$. The most remarkable feature is, however, the appearance of the preferential concentration in the case of $\operatorname{EXP} \beta$, similar to that observed in the case with larger particle inertia. ${ }^{13,54,55}$ Further discussion on the preferential concentration will be made in Sec. III E.

Significant contrast in the particle-settling pattern between EXP OA_ $\alpha$ and EXP LA_ $\alpha$ is observed in the distribution of particles in the vertical cross section at $t^{*}$ 

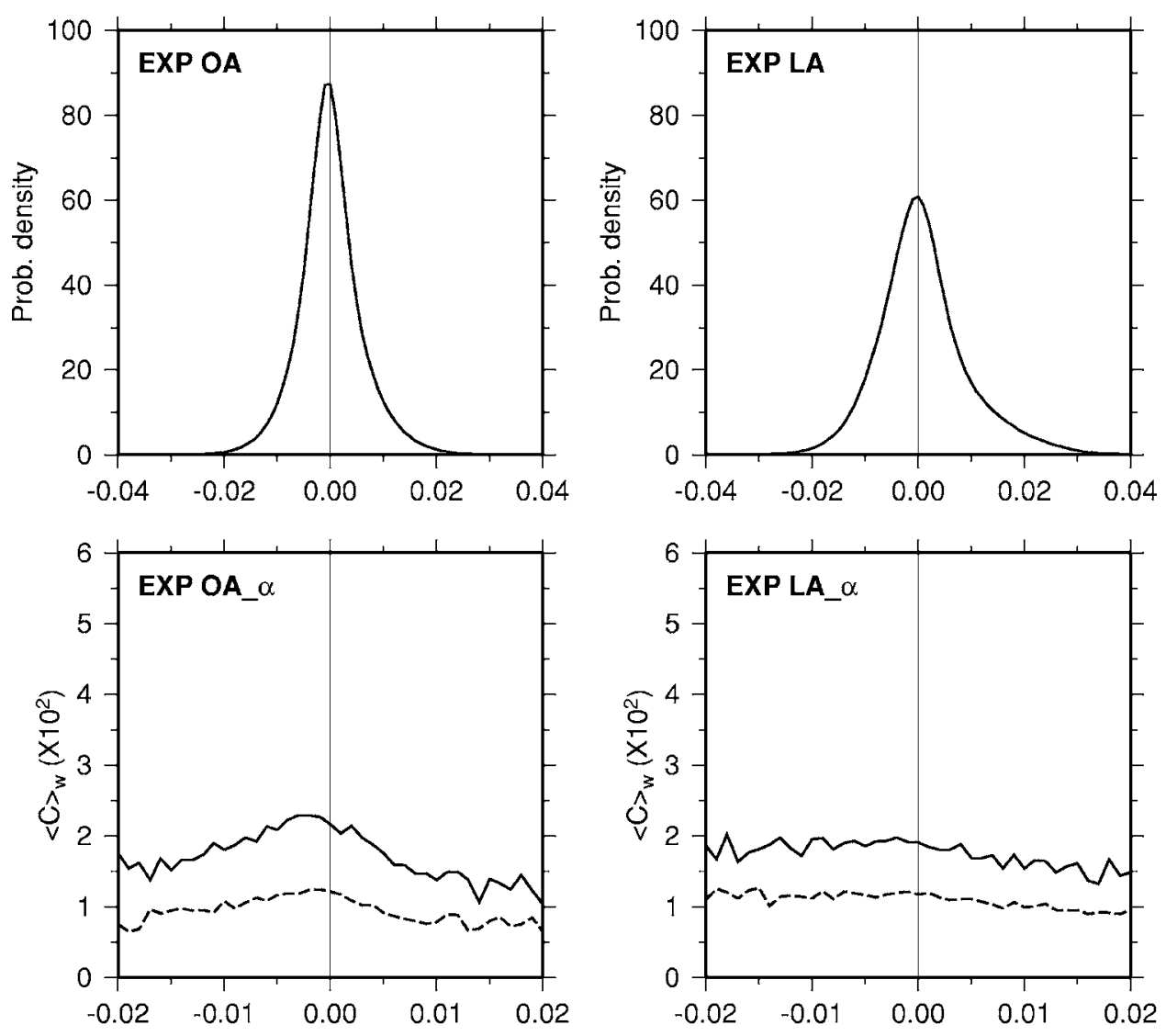

FIG. 7. Variation of the conditional averaged concentration of particles (EXP $\alpha$, middle; EXP $\beta$, bottom) with the vertical velocity together with the distribution of $w$ of the flow field (top). Here $\langle C\rangle_{w}$ was calculated by counting the number of particles in the grid points within the mixed layer $(z$ $<h)$ that have a given value of $w$ with the band of thickness $\Delta w=2.5$ $\times 10^{-3} \mathrm{~s}^{-1}$, and dividing it by the number of corresponding grid points (-, $t^{*}=0.8 ;---, t^{*}=1.2$ ).
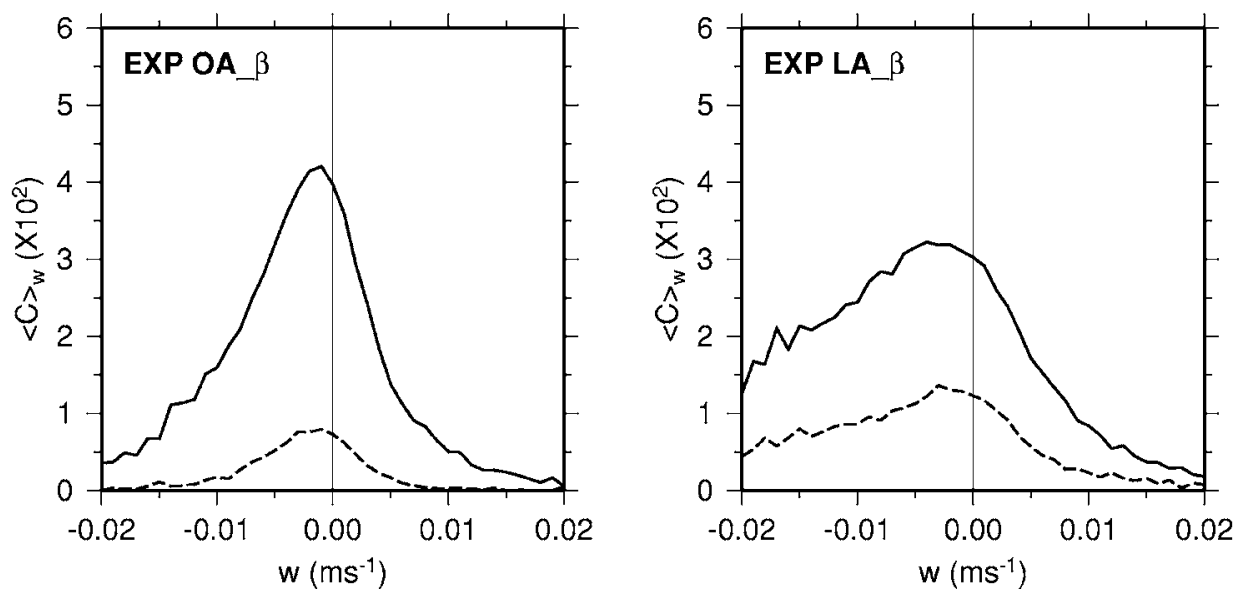

$=0.04$ (Fig. 2). In the absence of LC (EXP OA_ $\alpha)$, the downward transport of particles occurs more or less uniformly in the horizontal direction. On the other hand, in the presence of LC, it is dominated by the sweeping of particles by the downward jets associated with LC. This suggests that the description of the downward transport of particles in terms of the one-dimensional turbulent diffusion process, such as in Lande and Wood, ${ }^{4}$ may not be appropriate in the presence of LC.

The discrepancy can be identified more clearly in the tracks of vertical positions of particles (Fig. 4). In EXP OA_ $\alpha$, particles are moved up and down by small-scale eddies, while settling down slowly overall. In EXP LA_ $\alpha$, however, particles are swept downward rapidly to the mixed layer depth, and then move up again close to the surface. The much larger length scale of vertical fluctuation in EXP LA_ $\alpha$, compared to EXP OA_ $\alpha$, signifies the importance of the role played by large-scale vortices of LC.

In the case of $\operatorname{EXP} \beta$, the equivalent contrast between EXP OA and EXP LA, shown in Figs. 2 and 4, still appears, although it is much weaker because the settling process is dominated by the gravitational force (not shown).

\section{Evolution of the mean concentration of particles}

From the contrasting patterns of particle settling shown in Figs. 2 and 4, we can expect much faster downward transport of particles in the presence of LC, and this is confirmed in the evolution of the horizontal mean concentration profiles 

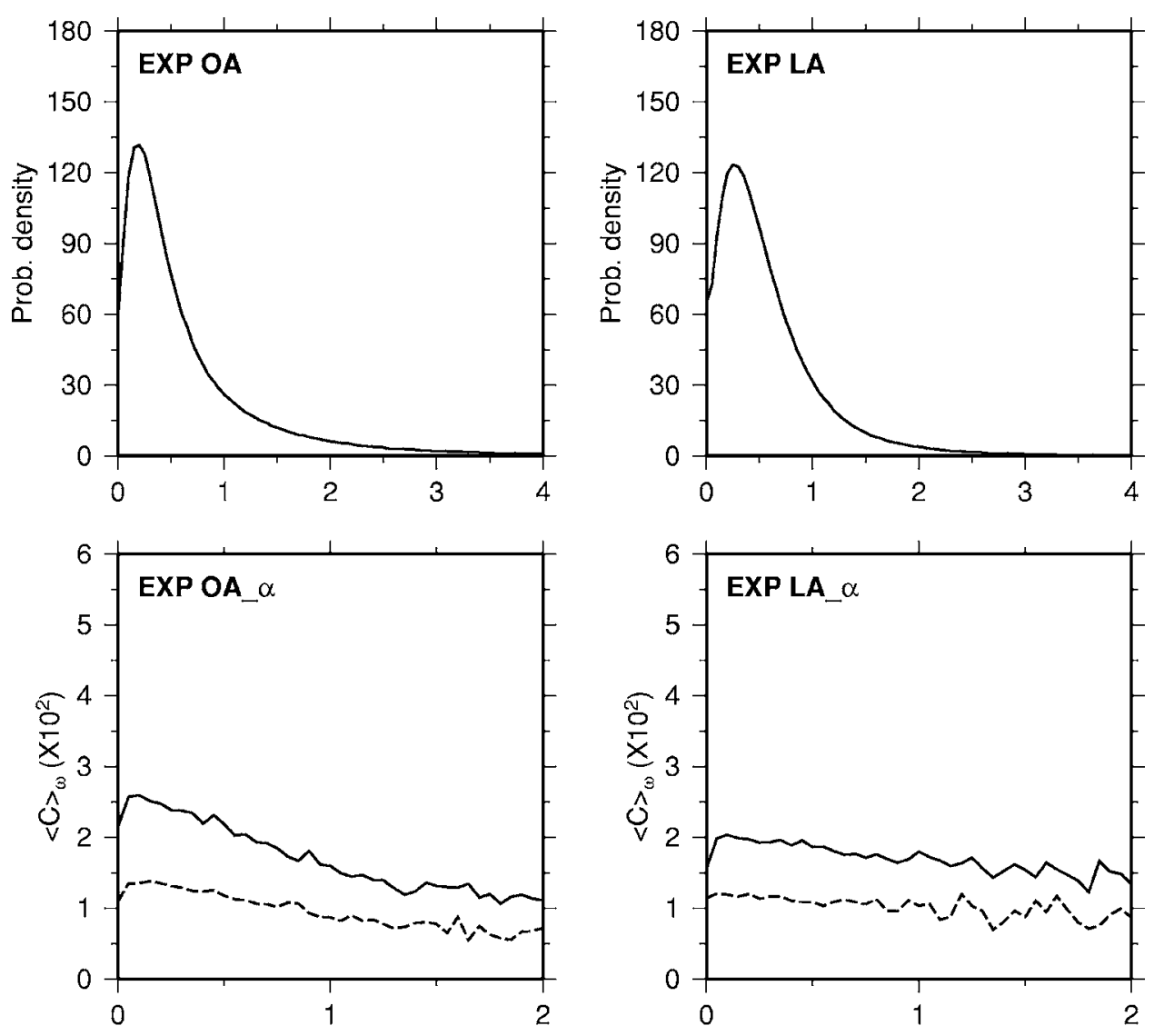

FIG. 8. Variation of the conditional averaged concentration of particles (EXP $\alpha$, middle; $\operatorname{EXP} \beta$, bottom) with the horizontal vorticity $\omega_{h} \quad\left(=\left[\omega_{x}^{2}\right.\right.$ $\left.+\omega_{y}^{2}\right]^{1 / 2}$ ) together with the distribution of $\omega_{h}$ of the flow field (top). Here $\langle C\rangle_{\omega}$ was calculated by counting the number of particles in the grid points within the mixed layer $(z<h)$ that have a given value of $\omega_{h}$ with the band of thickness $\Delta \omega_{h}=5 \times 10^{-4} \mathrm{~s}^{-1}$, and dividing it by the number of corresponding grid points $\left(-, t^{*}=0.8\right.$; ---, $\left.t^{*}=1.2\right)$.
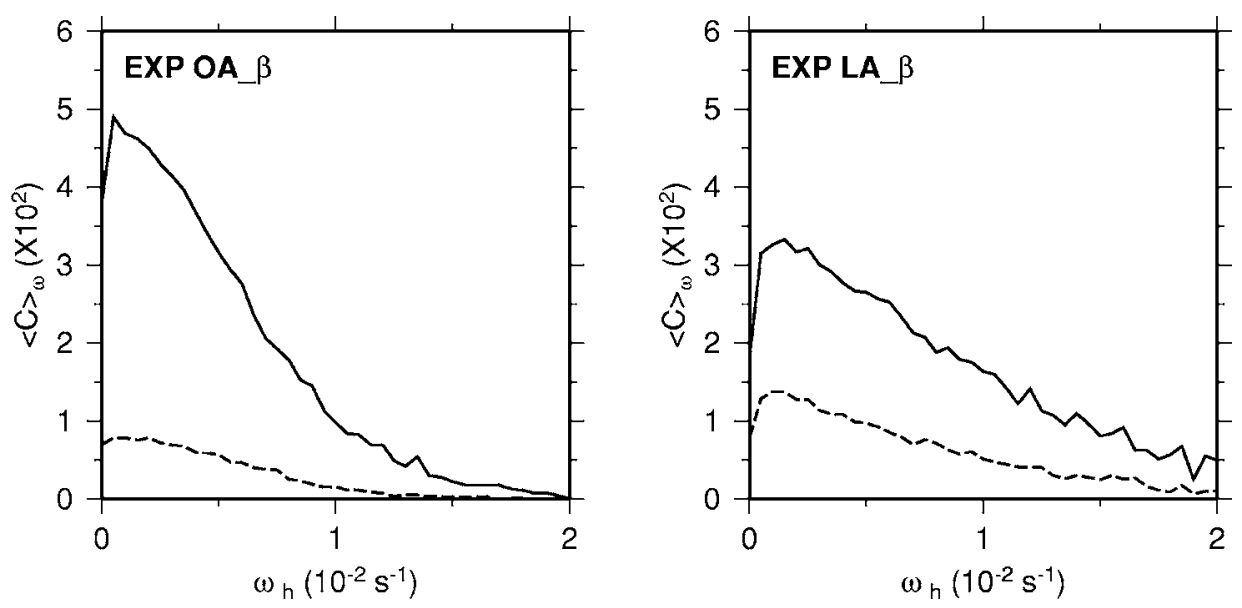

$\bar{C}(z)$ from EXP OA_ $\alpha$ and EXP LA_ $\alpha$ (Fig. 5). Here the mean concentration $\bar{C}(z)$ was obtained by the number of particles $N$ located within the vertical band of thickness $2 \mathrm{~m}$ for a given depth divided by the total number of released particles $N_{0}$.

In EXP LA_ $\alpha$, the depth of the maximum concentration propagates downward rapidly and an almost uniform concentration appears over the whole mixed layer at $t^{*} \sim 0.2$. The concentration $\bar{C}(z)$ decreases uniformly at all depths with time thereafter. On the other hand, in EXP OA_ $\alpha$, both the downward propagation of the depth of the maximum concentration and the diffusion of the concentration profile are slower, and a uniform concentration profile within the mixed layer cannot be obtained. This implies that vertical mixing becomes much stronger in the presence of LC.

\section{Settling velocity of particles}

Figure 6 shows the variation of the mean settling velocity $\langle W\rangle$ with time for each experiment, in which $\langle W\rangle$ is obtained by averaging $W\left(=-V_{3}\right)$ of particles present within the mixed layer $(0<z<h)$. The case of a passive tracer is also shown for comparison. In this case, we cannot rescale the velocity and time scales in terms of $w_{s}=0 \mathrm{~ms}^{-1}$, so we rescaled them by $w_{s}=10^{-3} \mathrm{~ms}^{-1}$ instead for the sake of comparison with the corresponding case $\left(\mathrm{EXP}_{-} \alpha\right)$.

Soon after the release of particles, $\langle W\rangle$ increases rapidly 

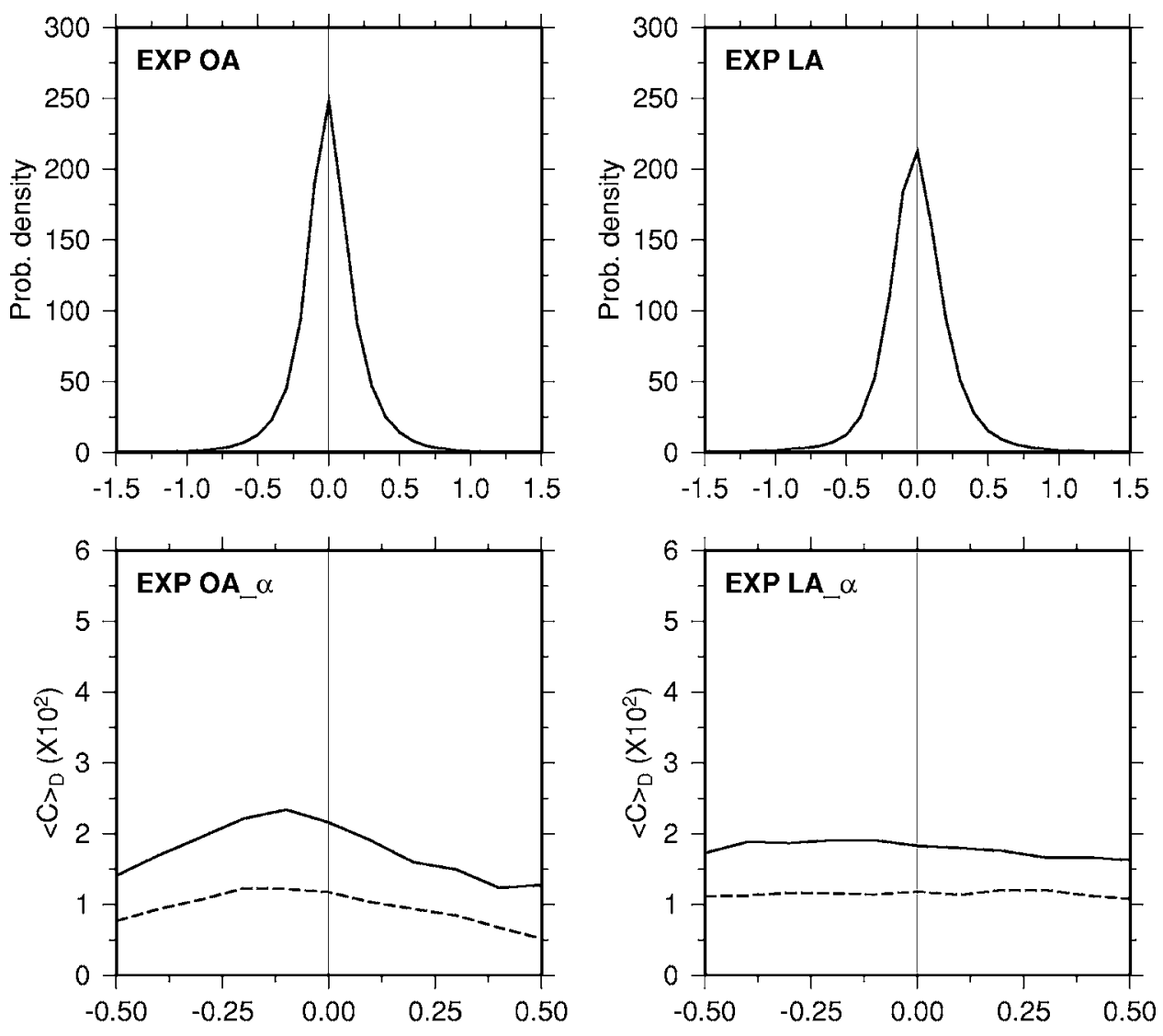

FIG. 9. Variation of the conditional averaged concentration of particles (EXP $\alpha$, middle; $\operatorname{EXP} \beta$, bottom) with the horizontal divergence $D_{h}(=\partial u / \partial x$ $+\partial v / \partial y)$ together with the distribution of $D_{h}$ of the flow field (top). Here $\langle C\rangle_{D}$ was calculated by counting the number of particles in the grids points within the mixed layer $(z<h)$ that have a given value of $D_{h}$ with the band of thickness $\Delta D_{h}=10^{-3} \mathrm{~s}^{-1}$, and dividing it by the number of corresponding grid points $\left(-, t^{*}=0.8 ;---, t^{*}=1.2\right)$.
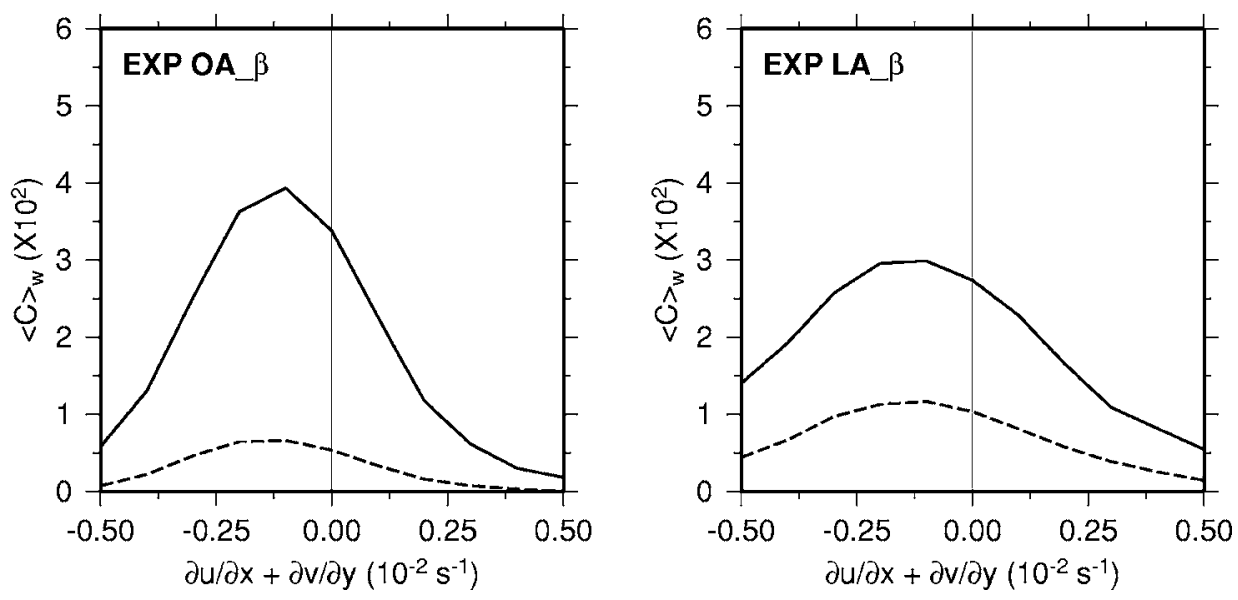

initially, primarily because most particles are present in the downward eddies during the initial downward propagation of the particle concentration front. It is also found that the initial increase of $\langle W\rangle$ is larger in the presence of $\mathrm{LC}$, and for smaller $w_{s}$. This effect diminishes with the lapse of time, and it becomes negligible after $t^{*} \sim 1$, as we can infer from the settling velocity of a passive tracer. The effect of particle reflection at the surface is negligible in the present simulation because of very small particle inertia.

The settling velocity $\langle W\rangle$ is smaller than $w_{s}$ in all experiments, and the magnitude of decrease is larger for smaller $w_{s}$. Meanwhile, it is found that $\langle W\rangle$ is further decreased in the presence of LC. In the case of EXP LA_ $\alpha,\langle W\rangle$ decreases to as much as $\langle W\rangle / w_{s}=0.55$. The decrease of $W$ in turbulent flows was also observed by Murray ${ }^{11}$ and Fung. ${ }^{12}$

\section{E. Relation between the particle concentration and the flow field}

With an aim to understanding the mechanism for the decrease of the settling velocity, we investigated the conditionally averaged concentration of particles for given values of vertical velocity $w$, horizontal divergence/convergence, $D_{h}$ $(=\partial u / \partial x+\partial v / \partial y), \quad$ and horizontal vorticity, $\omega_{h} \quad\left(=\left[\omega_{x}^{2}\right.\right.$ $\left.+\omega_{y}^{2}\right]^{1 / 2}$ ) at $t^{*}=0.8$ and 1.2 (Figs. 7-9).

Figure 7 shows that the particle concentration is higher in the region of upward fluid velocity than in that of downward fluid velocity in all cases. It leads to the decrease of $W$, as shown in Fig. 6; that is, $\langle W\rangle<w_{s}$, if $\langle w\rangle<0$, from (7), where $\langle w\rangle$ is the averaged vertical velocity of the fluid over all particle positions. This means that a particle spends more 


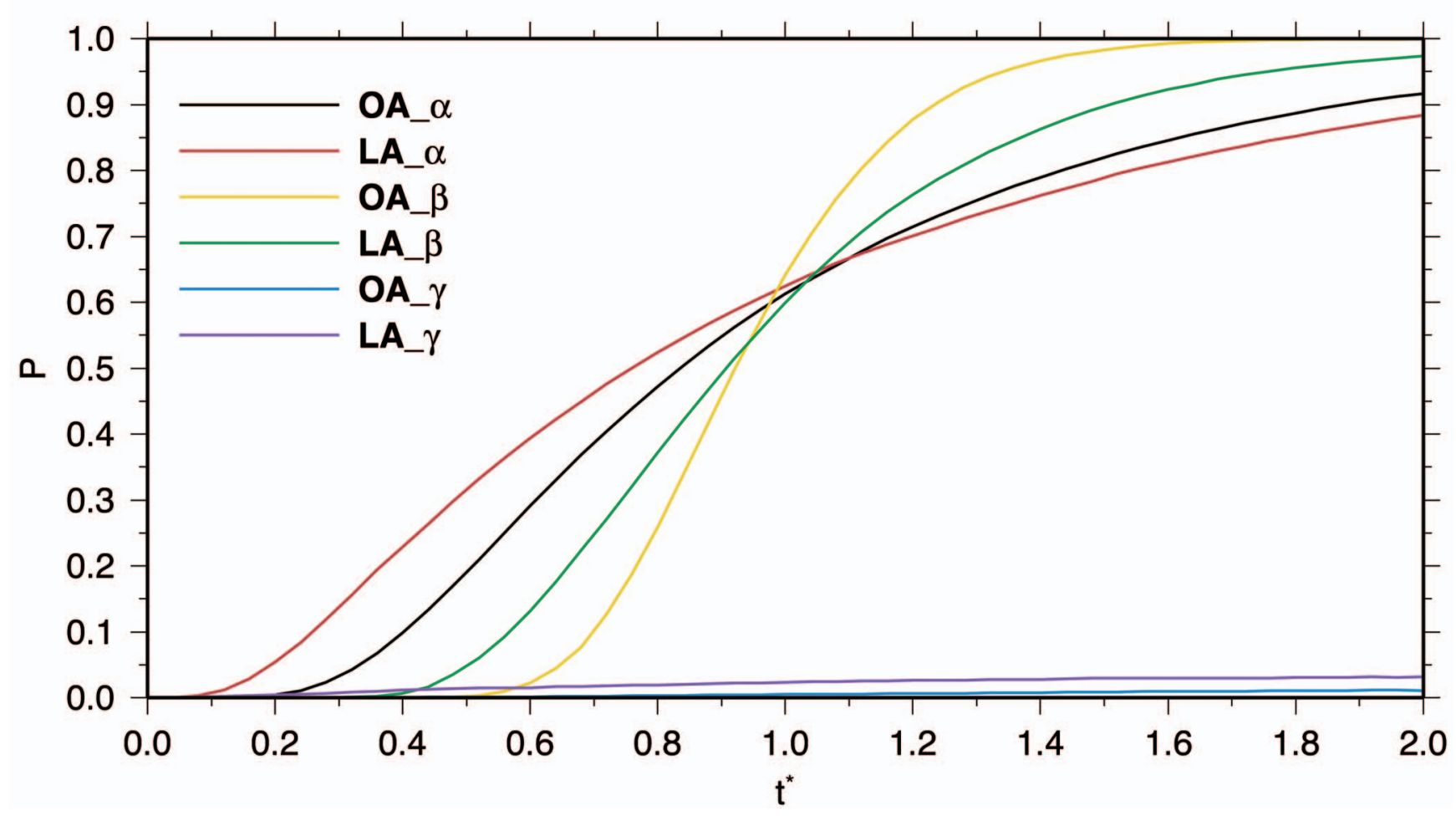

FIG. 10. (Color) Variation of the percentage of particles sinking below the mixed layer with time $t^{*}\left[=t /\left(h / w_{s}\right)\right]$ for each experiment. In the case of a passive tracer, both time and velocity scales were rescaled by using $w_{s}=10^{-3} \mathrm{~ms}^{-1}$.

time in upward flows than in downward flows in a statistical sense, if the steady state is assumed, similarly to the case of particle trapping within a vortex. ${ }^{5}$

Asymmetry of the particle concentration between the upward and downward fluid velocity is much stronger for EXP $\beta$ than for EXP $\alpha$, but the decrease of $\langle W\rangle / w_{s}$ is larger for $\operatorname{EXP} \alpha$. This may be due to the fact that particles interact with the flow field over a much longer time during settling for EXP $\alpha$, thus causing the larger modification of $\langle W\rangle / w_{s}$.

Note also that asymmetry with positive skewness appears in the distribution of the vertical velocity of the flow field in EXP LA, reflecting the existence of strong downward jets associated with LC (see Figs. 1 and 2), contrary to the symmetric distribution in EXP OA (Fig. 7).

Figures 8 and 9 show clearly that the particle concentration tends to decrease with increasing $\omega_{h}$ and $D_{h}$, and the tendency is much stronger in $\operatorname{EXP} \beta$ than in $\operatorname{EXP} \alpha$, which is realized by the particle distribution in Fig. 1. According to Eaton and Fessler, ${ }^{56}$ the preferential particle concentration occurs away from the regions of higher vorticity and divergence as a result of the centrifugal force, and it is the most significant when the time scales of turbulent eddies and particles inertia are equivalent; i.e., $\mathrm{St} \sim 1$. In the present simulation, $\mathrm{St}$ is very small $\left(\mathrm{St} \sim 10^{-4}\right)$. However, the ratio of the time scale of turbulent eddies to that for a particle to traverse an eddy, which can be represented by $w_{s} / u_{*}$, is about 1 in the case of EXP $\beta$, and it may also induce the preferential concentration by increasing the response to vortex motions, as suggested by Eaton and Fessler. ${ }^{56}$ It also clarifies why the case with $w_{s} / u_{*}=1$ (EXP $\beta$ ) shows much stronger preferential concentration than the case with $w_{s} / u_{*}=0.1(\operatorname{EXP} \alpha)$.
Meanwhile, the comparison between EXP OA and EXP LA reveals that more particles tend to accumulate in the high vorticity region in the presence of LC. It can be interpreted that particles are more likely to be trapped within vortices in the presence of LC, and it may lead to the decrease of $W$ in the presence of LC. In the present simulation, however, vortices vary rapidly with time and do not have well-defined closed paths, so that it is impossible to identify the trapping of particles explicitly.

The extension of Stommel's theory ${ }^{5}$ to the case of the Rankine vortex predicts that the trapping of particles with no inertia within a vortex is possible only if

$$
w_{s}<\omega R / 2 \text {, }
$$

where $\omega$ and $R$ are the vorticity and the radius of a Rankine vortex (see, e.g., Nielson ${ }^{49}$ ). We can expect from (8) that particle trapping is more likely when the length scale of vortices is larger in the presence of LC. The increase of the length scale in the presence of LC can be confirmed from the spectrum of the velocity field, as shown in Noh et al. ${ }^{27}$

Note that the preferential concentration toward the region of low vorticity does not lead to the increase of $\langle W\rangle$ in the present simulation, contrary to the previous experiments using isotropic turbulence. ${ }^{13-17}$ The most important difference of the present experiment from the previous ones is the length scale of turbulent eddies. It is an order of $10 \mathrm{~m}$ in the present simulation of the ocean mixed layer (see, e.g., Fig. 3 in Noh $e t a l .{ }^{27}$ ), whereas it was only a few $\mathrm{cm}$ in the isotropic turbulence of previous experiments. ${ }^{13-17}$ If the size of eddies becomes too small, it may not allow particle trapping, as expected from (8). It is expected that, in the present simu- 
lation with large vortices, the effect of particle trapping dominates over that of preferential concentration.

\section{F. Particle flux to below the mixed layer}

Figure 10 shows the variation of the probability of particles passing through the mixed layer depth $P[=1$ $\left.-\int_{0}^{h} \bar{C}(z) d z\right]$ with time. The increase of the mixed layer depth during experiments was negligible, with the largest increase of about $1 \mathrm{~m}$ in the case of EXP B. Note that all particles must pass through the depth $z=h$ when $t^{*}=1$ so as to make $P=1$ in the still fluid.

As expected from the decreased particle settling velocity, particles stay longer within the mixed layer in the presence of LC, although the initial particle flux is much larger due to the sweeping down by downward jets of LC. Meanwhile, the particle flux decreases with the decrease of $w_{s} / u *$.

Figure 10 also implies that $W$ of each particle has much larger variance in the presence of LC, which is consistent with Pasquero et al.'s result ${ }^{10}$ that the distribution of $W$ is significantly broadened in the presence of the spatial and temporal correlation of the flow, compared to the case with a white-noise spectrum, although the rms velocity is the same.

\section{G. Effects of the wind stress and a thermocline}

For the practical prediction of particle settling in the ocean mixed layer, we may need the information on the variations of $\langle W\rangle / w_{s}$ and $P$ in terms of the characteristics of particles and flow fields; for example, $w_{s}$ and $\tau_{p}$ of particles, and the wind stress and the buoyancy flux at the sea surface, the mixed layer depth, the vertical profiles of velocity and density within the mixed layer, the condition of surface gravity waves, etc. In the present study, we considered only the idealized situation without the surface buoyancy, and investigated how $\langle W\rangle / w_{s}$ and $P$ are affected by the wind stress and the presence of a thermocline.

Figure 11 shows that the settling velocity decreases, thus suppressing the particle flux further with decreasing $w_{s} / u *$. Here $\langle W\rangle$ was obtained by averaging the value during $t^{*}$ $=1-2$, since $W$ approaches the steady state near $t^{*}=1$ (Fig. 6). The particle flux $P^{*}$ was obtained at $t^{*}=1.6$, at which the difference of $P$ tends to be the largest (Fig. 10). The values of $\langle W\rangle$ and $P^{*}$ are always smaller in the presence of $\mathrm{LC}$, but the difference tends to be the largest at $w_{s} / u * \sim 1$, where the strongest correlation exists between particles and the flow field (Figs. 7-9). Considering the tendency of decreasing settling velocity and particle flux with $w_{s} / u^{*}$, we can expect even smaller values of $\langle W\rangle / w_{s}$ and $P^{*}$ for particles for smaller $w_{s} / u^{*}$.

Especially, the comparison of Figs. 11 and 12 makes it clear that the presence of a large-scale coherent vortex of LC suppresses particle settling, even if the level of TKE is equivalent. The vertical profile of TKE shows that the TKE of EXP OB is generally larger than that of EXP LA (Fig. 12), but $\langle W\rangle / w_{s}$ is much smaller in EXP LA (Fig. 11). Likewise, the TKE of EXP LC is much smaller than that of EXP OA, but the values of $\langle W\rangle / w_{s}$ are comparable.

Finally, we examined the case with a homogeneous
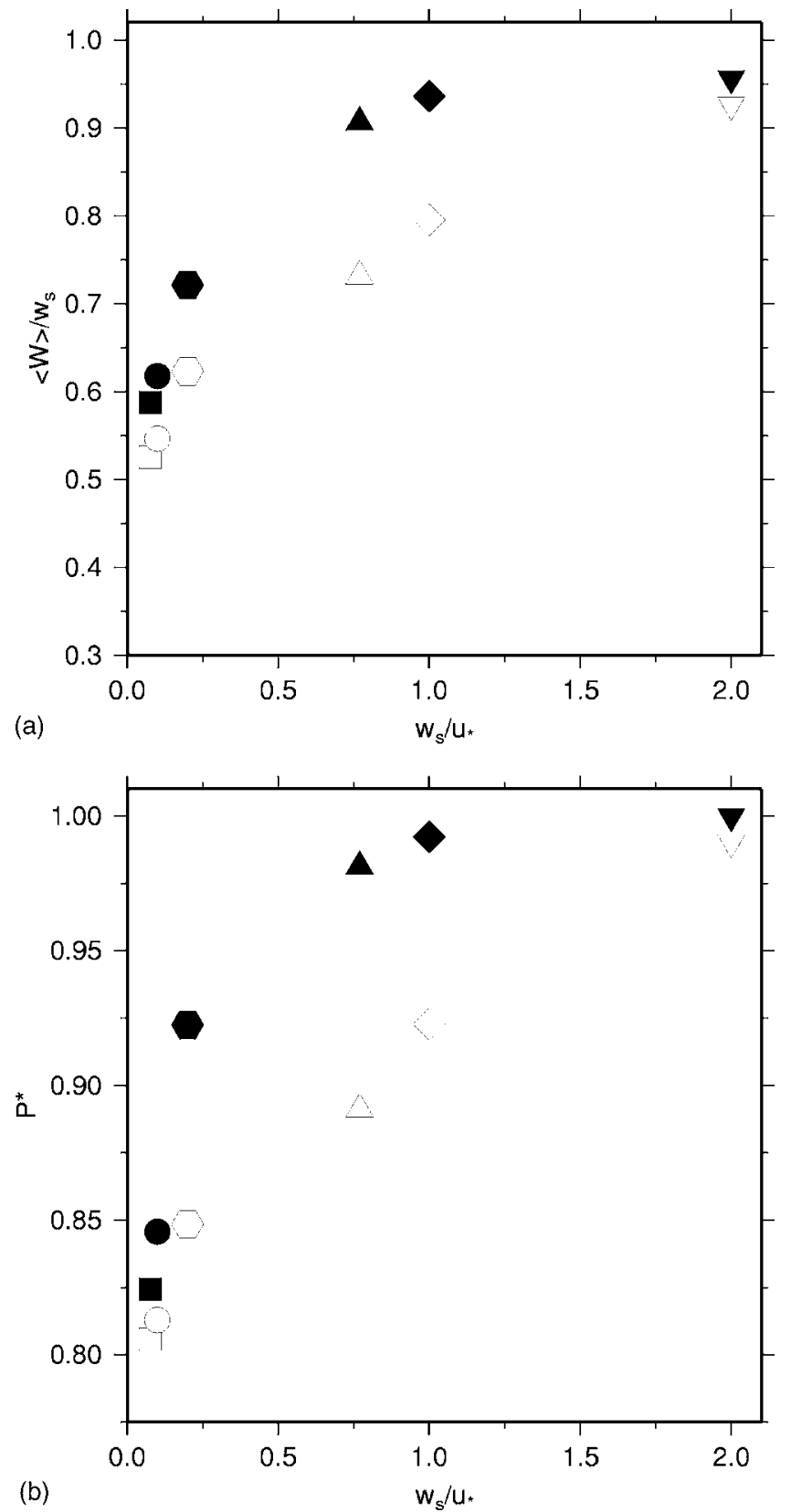

FIG. 11. (a) Variation of $\langle W\rangle / w_{s}$ with $w_{s} / u^{*}$. (b) Variation of $P^{*}\left[=P\left(t^{*}\right.\right.$ $=1.6)]$ with $w_{s} / u^{*}$. ( $\left(\mathrm{OA}_{-} \alpha, \boldsymbol{\square}\right.$ : OB_$\alpha$, black octagon: $\mathrm{OC}_{-} \alpha, \bullet: \mathrm{OA}_{-} \beta$, $\boldsymbol{\Delta}: \mathrm{OB}_{-} \beta, \boldsymbol{\nabla}: \mathrm{OC}_{-} \beta, \mathrm{O}_{\mathrm{L}} \mathrm{LA}_{-} \alpha, \square: \mathrm{LB}_{-} \alpha$, open octagon: LC_ $\alpha, \diamond: \mathrm{LA}_{-} \beta$ $\triangle: \mathrm{LB}_{-} \beta, \nabla: \mathrm{LC}_{-} \beta$.

mixed layer in order to investigate the effect of a finite thickness of the ocean mixed layer bounded by a thermocline. For the comparison with EXP A, we assumed an imaginary mixed layer depth at $z=h(=50 \mathrm{~m})$; that is, $\langle W\rangle$ was calculated by averaging the particles within $z<h$, and $P^{*}$ was calculated by counting the particles passing through $z<h$.

Since turbulent eddies and LC are not interrupted by a thermocline in the homogeneous layer (EXP D), they penetrate deeper and remain stronger with increasing depth (Fig. 13). As a result, in EXP D, the initial downward propagation of particles is faster (not shown), but particle settling ultimately becomes slower. It also implies that the influence of 

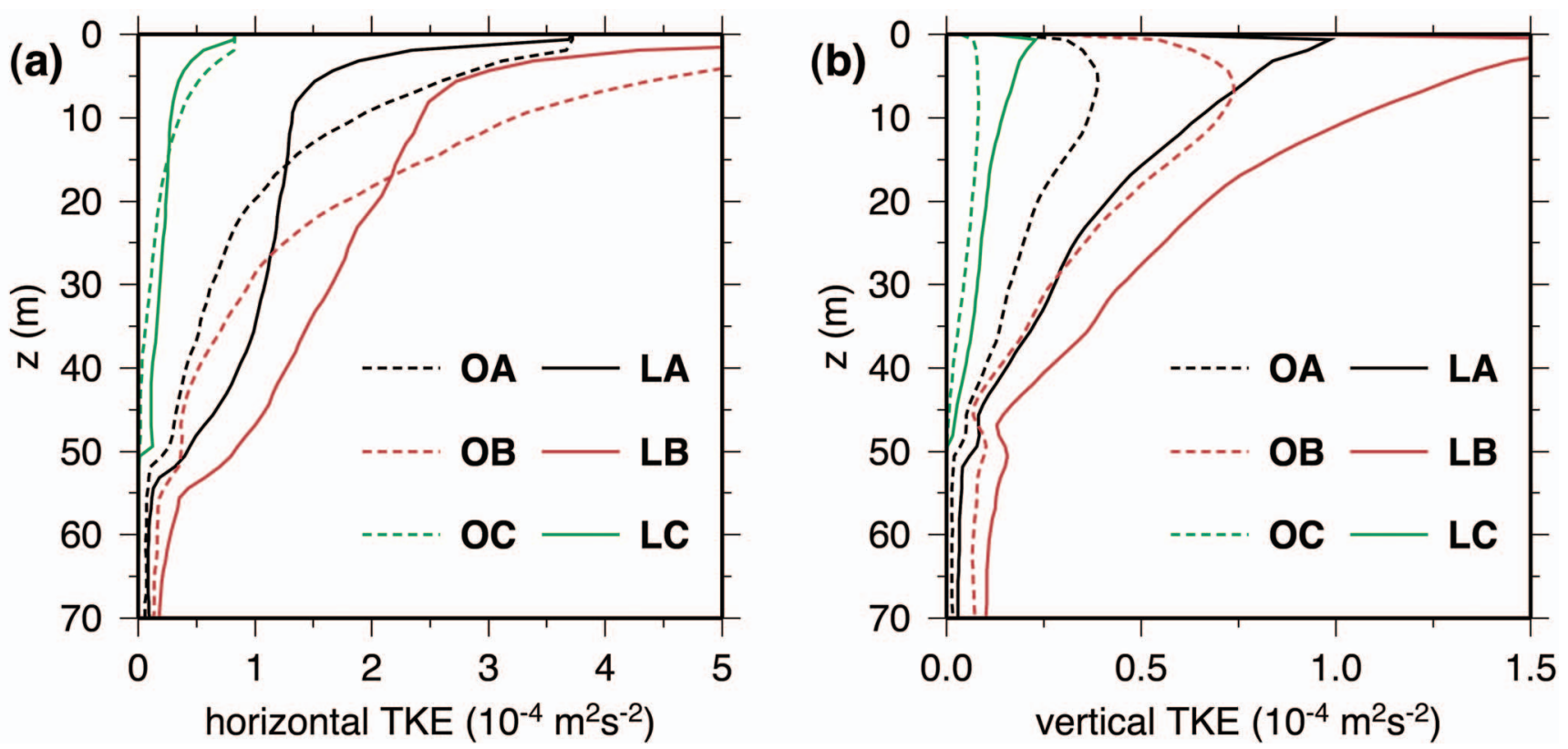

FIG. 12. (Color) Profiles of the mean horizontal TKE (a) and the vertical TKE (b).

the mixed layer turbulence to suppress the particle settling is stronger when the mixed layer depth is larger.

In Fig. 14, the values of $\langle W\rangle / w_{s}$ of EXP OD_ $\beta$ and EXP $\mathrm{LD}_{-} \beta$ do not represent correctly the actual particle settling velocity, because particles used to calculate $\langle W\rangle / w_{s}$ here are mainly located within the upward moving part of eddies in the absence of a thermocline, after most particles fall below $z=h$ at large $t^{*}$. The values of $\langle W\rangle / w_{s}$ are observed to continue to decrease with time in this case (not shown).

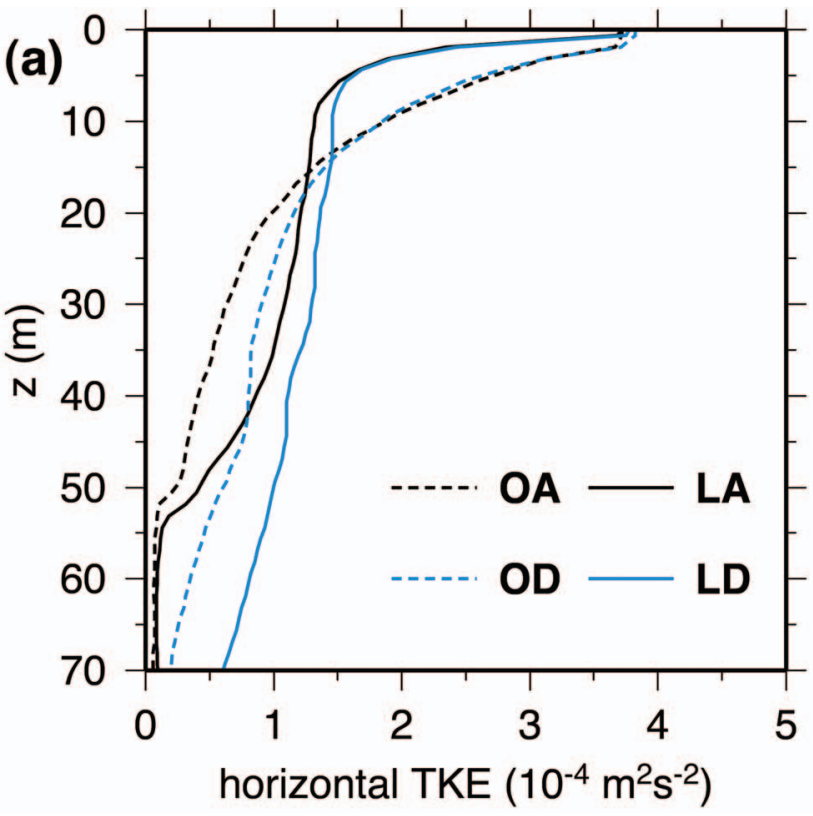

\section{CONCLUSION}

In the present work, we calculated for the first time the settling velocity of suspended particles in the ocean mixed layer, by analyzing the Lagrangian motion of a large number of particles in the turbulent flow field simulated by LES. Investigation was focused on how the presence of large-scale coherent vortices of Langmuir circulation (LC) affects the particle settling.

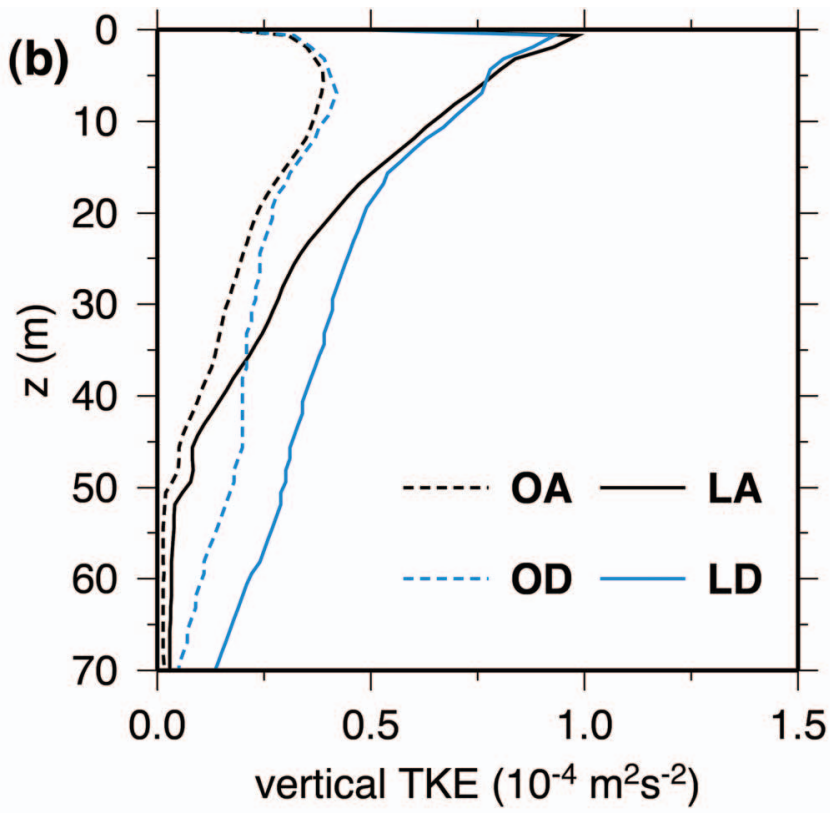

FIG. 13. (Color) (a) Variation of $\langle W\rangle / w_{s}$ with $w_{s} / u^{*}$. (b) Variation of $P^{*}\left[=P\left(t^{*}=1.6\right)\right]$ with $w_{s} / u^{*}$. $\square: L_{-} \alpha, \triangle: \mathrm{OD}_{-} \beta, \nabla: \mathrm{LD}_{-} \beta$.) 


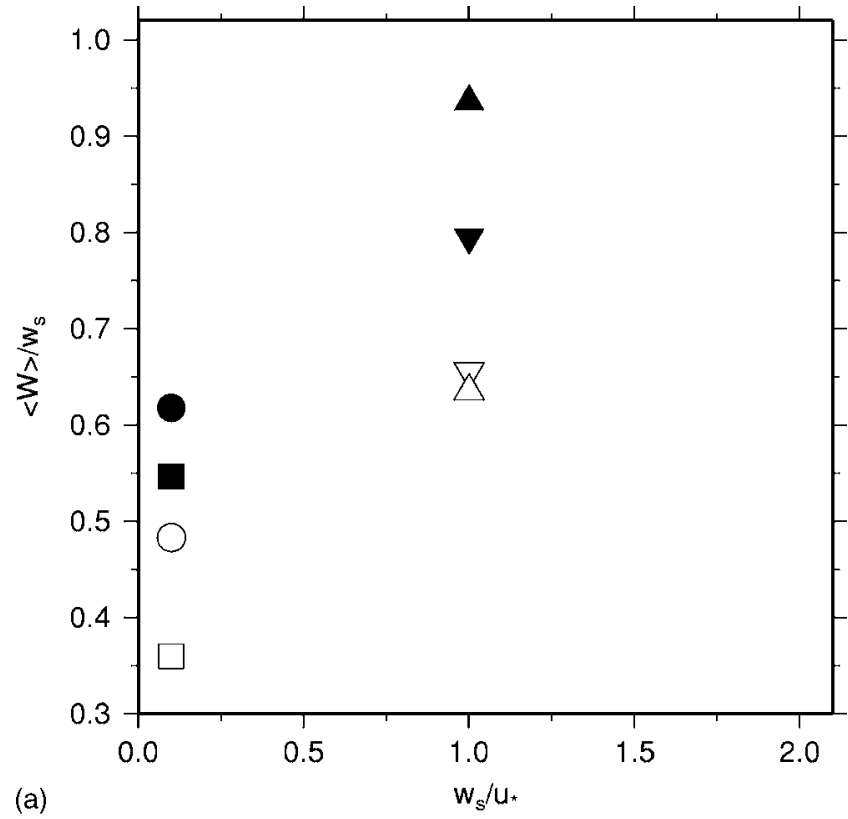

(a)

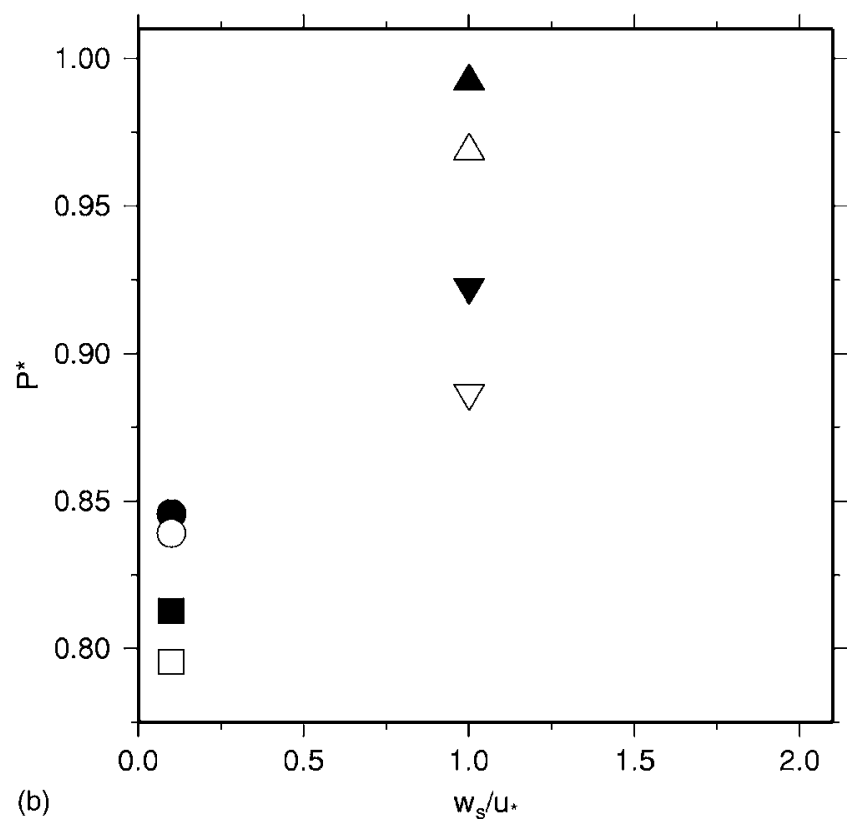

FIG. 14. Profiles of the mean horizontal TKE (a) and the vertical TKE (b).

At the beginning, particles released near the surface are swept down rapidly following the downward jets of LC in the presence of LC, contrary to the case of the horizontally uniform downward propagation in the absence of it. It suggests that the description of the downward transport of particles in terms of the one-dimensional turbulent diffusion process may not be appropriate in the presence of LC. The strong vertical mixing by LC keeps the particle concentration almost uniform after the initial period in the presence of LC, while its mean concentration continues to decrease through particle settling.

Under the influence of turbulence in the ocean mixed layer, the particle settling velocity $W$ is always smaller than $w_{s}$, and it decreases with decreasing $w_{s} / u^{*}$, where $w_{s}$ is the terminal velocity of a particle in the still fluid and $u *$ is the frictional velocity. The value of $W$ is decreased further in the presence of LC, although the initial downward propagation is much faster. The difference between the cases with and without LC is the largest at $w_{s} / u * \sim 1$. It was also found that $W$ becomes smaller in the homogeneous mixed layer without a thermocline, because LC is not interrupted by a thermocline.

The present result substantiates that the presence of a realistic large-scale coherent vortex motion such as LC can actually suppress the particle settling in the turbulent boundary layer, which has been a subject of conjecture for a long time, ${ }^{3,4}$ since Stommel ${ }^{5}$ showed that particles can be trapped indefinitely in a stationary laminar vortex.

The analysis of LES data reveals that particles spend more time in upward flows and more particles tend to accumulate in the high vorticity region in the presence of LC. From the condition of the trapping of particles with no inertia within the Rankine vortex such as $w_{s}<\omega R / 2$, where $\omega$ and $R$ are the vorticity and the radius of the vortex, we can expect that particle trapping is more likely when the length scale of vortices is larger, and it may lead to the decrease of $W$ in the presence of LC. Although the preferential concentration away from the regions of high horizontal vorticity and divergence is observed when $w_{s} / u * \sim 1$, it does not lead to the decrease of $W$, contrary to the case of previous experiments with isotropic turbulence. ${ }^{13-17}$ This is probably because of the much larger length scale of turbulent eddies in the present simulation, which allows particle trapping within vortices. Further analysis will be required in the future, however, in order to clarify the mechanism. It will also be interesting to investigate how the particle settling process is affected by other coherent vortices in turbulent flows or by the modification of the turbulence spectrum.

The present paper provides information on the particle settling velocity and the particle flux in terms of $w_{s} / u^{*}$ in the ideal case without the surface buoyancy flux. More extensive experiments, taking into account various other conditions of particles and flow fields, are required, however, to provide the practical prediction. For example, the generation of convection under the surface cooling is expected to modify the particle settling process significantly not only by changing the turbulence structure of the mixed layer, but also by increasing the mixed layer depth rapidly.

\section{ACKNOWLEDGMENTS}

This work was supported by the Korea Research Foundation (KRF-2003-015-C00684), KOSEF (International Cooperative Research Program), the supercomputing resources of the KISTI, and by the German Research Foundation (DFG) under Grant No. RA 617/7-1.

\footnotetext{
${ }^{1}$ Particle Flux in the Ocean, edited by V. Ittekkot, P. Schäfer, S. Honjo, and P. J. Depetris (John Wiley \& Sons, Chichester, 1996).

${ }^{2}$ Ocean Biogeochemistry, edited by M. J. R. Fasham (Springer Verlag, Berlin, 2003).

${ }^{3}$ K. L. Denman and A. E. Gargett, "Biological-physical interactions in the upper ocean: The role of vertical and small scale transport process," Annu. Rev. Fluid Mech. 27, 225 (1995)

${ }^{4}$ R. Lande and A. M. Wood, "Suspension times of particles in the upper ocean,” Deep-Sea Res., Part A 34, 61 (1987).

${ }^{5} \mathrm{H}$. Stommel, "Trajectories of small bodies sinking slowly through convection cells," J. Mar. Res. 8, 24 (1949).

${ }^{6}$ P. F. Tooby, G. L. Wick, and J. D. Isaacs, "The motion of a small sphere
} 
in a rotating velocity field: A possible mechanism for suspending particles in turbulence," J. Geophys. Res. 82, 2096 (1977).

${ }^{7}$ M. R. Maxey and S. Corrsin, "Gravitational settling of aerosol particles in randomly oriented cellular flow fields," J. Atmos. Sci. 43, 1112 (1986).

${ }^{8}$ J. Dávila and J. C. R. Hunt, "Settling of small particles near vortices and in turbulence," J. Fluid Mech. 440, 117 (2001).

${ }^{9}$ I. Eames and M. A. Gilbertson, "The settling and dispersion of small dense particles by spherical vortices," J. Fluid Mech. 498, 183 (2004).

${ }^{10}$ C. Pasquero, A. Provenzale, and E. A. Spiegel, "Suspension and fall of heavy particles in random two-dimensional flow," Phys. Rev. Lett. 91, 054502 (2003)

${ }^{11}$ S. P. Murray, "Settling velocities and vertical diffusion of particles in turbulent water," J. Geophys. Res. 75, 1647 (1970).

${ }^{12}$ J. C. H. Fung, "Gravitational settling of particles and bubbles in homogeneous turbulence,” J. Geophys. Res. 98, 20287 (1993).

${ }^{13}$ L. P. Wang and M. R. Maxey, "Settling velocity and concentration distribution of heavy particles in homogeneous isotropic turbulence," J. Fluid Mech. 256, 27 (1993).

${ }^{14}$ M. R. Maxey, B. K. Patel, E. J. Chang, and L. P. Wang, "Simulations of dispersed turbulent multiphase flow," Fluid Dyn. Res. 20, 143156 (1997).

${ }^{15}$ Y. Yang and U. Lei, "The role of the turbulent scales in the settling velocity of heavy particles in homogeneous isotropic turbulence," J. Fluid Mech. 371, 179 (1998).

${ }^{16}$ A. Aliseda, A. Cartellier, F. Hainaux, and J. C. Lasheras, "Effects of preferential concentration on the settling velocity of heavy particles in homogeneous isotropic turbulence," J. Fluid Mech. 468, 77 (2002).

${ }^{17}$ T. S. Yang and S. S. Shy, "The settling velocity of heavy particles in an aqueous near-isotropic turbulence," Phys. Fluids 15, 868 (2003).

${ }^{18}$ G. W. Slack, "Sedimentation of compact clusters of uniform spheres," Nature (London) 200, 466 (1963).

${ }^{19}$ Y. Noh and H. J. S. Fernando, "The transition in the sedimentation pattern of a particle cloud," Phys. Fluids A 5, 3049 (1993).

${ }^{20}$ Y. Noh, "Sedimentation of a particle cloud across a density interface," Fluid Dyn. Res. 27, 129 (2000).

${ }^{21}$ S. Leibovich, "The form and dynamics of Langmuir circulation," Annu. Rev. Fluid Mech. 15, 391 (1983).

${ }^{22}$ R. A. Weller and J. F. Price, "Langmuir circulation within the oceanic mixed layer," Deep-Sea Res., Part A 35, 711 (1988).

${ }^{23}$ A. J. Pluedemann, J. A. Smith, D. A. Farmer, R. A. Weller, W. R. Crawford, R. Pinkel, S. Vagle, and A. Gnanadesikan, "Structure and variability of Langmuir circulation during the surface waves process program," J. Geophys. Res. 101, 3525 (1996).

${ }^{24}$ A. D. D. Craik and S. Leibovich, "A rational model for Langmuir circulations," J. Fluid Mech. 73, 402 (1976).

${ }^{25}$ E. D. Skyllingstad and D. W. Denbo, "An ocean large-eddy simulation of Langmuir circulations and convection in the surface mixed layer," J. Geophys. Res. 100, 8501 (1995).

${ }^{26}$ J. C. McWilliams, P. P. Sullivan, and C. H. Moeng, "Langmuir turbulence in the ocean," J. Fluid Mech. 334, 1 (1997).

${ }^{27}$ Y. Noh, H. S. Min, and S. Raasch, "Large eddy simulation of the ocean mixed layer: The effects of wave breaking and Langmuir circulation," J. Phys. Oceanogr. 34, 720 (2004).

${ }^{28} \mathrm{~F}$. Yeh and U. Lei, "On the motion of small particles in a homogeneous isotropic turbulent flow," Phys. Fluids A 3, 2571 (1991).

${ }^{29} \mathrm{P}$. J. Mason, "Large-eddy simulation of dispersion in convective boundary layers with wind shear," Atmos. Environ., Part A 26, 1561 (1992).

${ }^{30}$ Q. Wang and K. D. Squires, "Large eddy simulation of particle-laden turbulent channel flows," Phys. Fluids 8, 1207 (1996).

${ }^{31}$ W. S. J. Uijttewaal and R. V. A. Oliemans, "Particle dispersion and deposition in direct numerical simulation and large eddy simulation," Phys. Fluids 8, 2590 (1996).

${ }^{32}$ Y. Yamamoto, M. Potthoff, T. Tanakak, T. Kajishima, and Y. Tsuji, "Largeeddy simulation of turbulent gas-particle flow in a vertical channel: Effect of considering inter-particle collisions," J. Fluid Mech. 442, 303 (2001).

${ }^{33}$ E. D. Skyllingstad, "The effects of Langmuir circulation on buoyant particles," in Handbook of Scaling Methods in Aquatic Ecology, edited by L. Seuront and P. G. Strutton (CRC, Boca Raton, FL, 2004), pp. 445-452.

${ }^{34}$ R. R. Harcourt, E. L. Steffen, R. W. Garwood, and E. A. D'Asaro, "Fully Lagrangian floats in Labrador Sea deep convection: Comparison of numerical simulation and experimental results," J. Phys. Oceanogr. 32, 493 (2002).

${ }^{35} \mathrm{~S}$. Raasch and G. Harbusch, "An analysis of secondary circulations and their effects caused by small-scale surface inhomogeneities using largeeddy simulation," Boundary-Layer Meteorol. 101, 31 (2001).

${ }^{36} \mathrm{~S}$. Weinbrecht and S. Raasch, "High resolution simulations of the turbulent flow in the vicinity of an arctic lead," J. Geophys. Res. 106, 27035 (2001).

${ }^{37}$ M. O. Letzel and S. Raasch, "Large eddy simulation of thermally induced oscillations in the convective boundary layer," J. Atmos. Sci. 60, 2328 (2003).

${ }^{38}$ Y. Noh, W. G. Cheon, S. Y. Hong, and S. Raasch, "Improvement of the K-profile model layer based on large eddy simulation data," BoundaryLayer Meteorol. 107, 401 (2003).

${ }^{39}$ S. Raasch and D. Etling, "Modeling deep ocean convection: Large eddy simulation in comparison with laboratory experiments," J. Phys. Oceanogr. 28, 1786 (1998).

${ }^{40}$ Y. Noh, W. G. Cheon, and S. Raasch, "The role of preconditioning on the onset of open-ocean deep convection," J. Phys. Oceanogr. 33, 1145 (2003).

${ }^{41}$ H. S. Min and Y. Noh, "Influence of the surface heating on Langmuir circulation," J. Phys. Oceanogr. 34, 2630 (2004).

${ }^{42}$ J. W. Deardorff, "Stratocumulus-capped mixed layers derived from a three-dimensional model," Boundary-Layer Meteorol. 18, 495 (1980).

${ }^{43}$ C. H. Moeng, "A large-eddy simulation of the convective boundary layer," J. Atmos. Sci. 41, 2052 (1984).

${ }^{44}$ F. T. M. Nieuwstadt and R. A. Brost, "The decay of convective turbulence," J. Atmos. Sci. 43, 532 (1986).

${ }^{45} \mathrm{H}$. Schumidt and U. Schumann, "Coherent structure of the convective boundary layer derived from large-eddy simulation," J. Fluid Mech. 200, 511 (1989).

${ }^{46} \mathrm{~S}$. Raasch and M. Schröter, "A large eddy simulation model performing on massively parallel computers," Z. Meteorol. 10, 363 (2001).

${ }^{47}$ J. Smith, "Observed growth of Langmuir circulation," J. Geophys. Res. 97, 5651 (1992).

${ }^{48}$ Y. Noh and H. J. S. Fernando, "Dispersion of suspended particles in turbulent flow," Phys. Fluids A 3, 1730 (1991).

${ }^{49} \mathrm{P}$. Nielson, Coastal and Boundary Layers and Sediment Transport (World Scientific, Singapore, 1992), pp. 161-200.

${ }^{50} \mathrm{~S}$. Thorpe, "The break-up of Langmuir circulation and the instability of an array of vortices," J. Phys. Oceanogr. 22, 350 (1992).

${ }^{51}$ D. M. Farmer and M. Li, "Patterns of bubble clouds organized by Langmuir circulation," J. Phys. Oceanogr. 25, 1426 (1995).

${ }^{52}$ G. L. Mellor and T. Yamada, "Development of a turbulent closure model for geophysical fluid problems," Rev. Geophys. Space Phys. 20, 851 (1982).

${ }^{53}$ Y. Noh, C. J. Jang, T. Yamagata, P. C. Chu, and C. H. Kim, "Simulation of more realistic upper-ocean processes from OGCM with a new ocean mixed layer model," J. Phys. Oceanogr. 32, 1284 (2002).

${ }^{54}$ K. D. Squire and J. K. Eaton, "Preferential concentration of particles by turbulence," Phys. Fluids A 3, 1169 (1991).

${ }^{55}$ B. van Haarlem and F. T. M. Nieuwstadt, "Direct numerical simulation of particle motion in relation to structures in convective boundary layer," in Developments in Geophysical Turbulence, edited by R. M. Kerr and Y. Kimura (Springer Verlag, Berlin, 2000), pp. 37-50.

${ }^{56}$ J. K. Eaton and J. R. Fessler, "Preferential concentration of particles by turbulence," Int. J. Multiphase Flow 20, Suppl. 169 (1994). 